ARTICLE

DOI: $10.1038 / s 41467-018-07341-4$

\title{
RET rearrangements are actionable alterations in breast cancer
}

Bhavna S. Paratala1,2, Jon H. Chung (10 3 ${ }^{3}$, Casey B. Williams ${ }^{4}$, Bahar Yilmazel ${ }^{3}$, Whitney Petrosky ${ }^{1,2}$, Kirstin Williams ${ }^{4}$, Alexa B. Schrock ${ }^{3}$, Laurie M. Gay ${ }^{3}$, Ellen Lee ${ }^{5}$, Sonia C. Dolfi ${ }^{1,2}$, Kien Pham ${ }^{6}$, Stephanie Lin ${ }^{1,2}$, Ming Yao ${ }^{1,2}$, Atul Kulkarni 1,2, Frances DiClemente ${ }^{1,2}$, Chen Liu6 ${ }^{6}$, Lorna Rodriguez-Rodriguez ${ }^{2,7}$, Shridar Ganesan ${ }^{1,2}$, Jeffrey S. Ross ${ }^{3}$, Siraj M. Ali ${ }^{3}$, Brian Leyland-Jones ${ }^{4}$ \& Kim M. Hirshfield ${ }^{1,2}$

Fusions involving the oncogenic gene RET have been observed in thyroid and lung cancers. Here we report RET gene alterations, including amplification, missense mutations, known fusions, novel fusions, and rearrangements in breast cancer. Their frequency, oncogenic potential, and actionability in breast cancer are described. Two out of eight RET fusions (NCOA4-RET and a novel RASGEFTA-RET fusion) and RET amplification were functionally characterized and shown to activate RET kinase and drive signaling through MAPK and PI3K pathways. These fusions and RET amplification can induce transformation of non-tumorigenic cells, support xenograft tumor formation, and render sensitivity to RET inhibition. An index case of metastatic breast cancer progressing on HER2-targeted therapy was found to have the NCOA4-RET fusion. Subsequent treatment with the RET inhibitor cabozantinib led to a rapid clinical and radiographic response. RET alterations, identified by genomic profiling, are promising therapeutic targets and are present in a subset of breast cancers.

\footnotetext{
${ }^{1}$ Department of Medicine, Division of Medical Oncology, Rutgers Cancer Institute of New Jersey, New Brunswick, NJ 08901, USA. ${ }^{2}$ Rutgers University, Piscataway, NJ 08854, USA. ${ }^{3}$ Foundation Medicine, Cambridge, MA 02139, USA. ${ }^{4}$ Avera Cancer Institute Center for Precision Oncology, Sioux Falls, SD 57105, USA. ${ }^{5}$ University Radiology Group, New Brunswick, NJ 08901, USA. ${ }^{6}$ Department of Pathology and Laboratory Medicine, Rutgers New Jersey Medical School and Rutgers Robert Wood Johnson Medical School, Newark, NJ 07103, USA. ${ }^{7}$ Department of Obstetrics and Gynecology, Division of Gynecologic Oncology, Rutgers Cancer Institute of New Jersey, New Brunswick, NJ 08901, USA. Correspondence and requests for materials should be addressed to B.L-J. (email: brian.leylandjones@avera.org) or to K.M.H. (email: kim.hirshfield@merck.com)
} 
O ncogenic RET (rearranged during transfection) gene fusions have been described in $6.8 \%$ of papillary thyroid (PTC) and 1-2\% of non-small cell lung cancers (NSCLC) 1,2 . Fusion-related structural alterations of RET, a transmembrane receptor tyrosine kinase, lead to ligand-independent dimerization and constitutive kinase activation in turn driving oncogenic signaling cascades and increased cell survival and proliferation ${ }^{3}$. Most RET fusions lack the N-terminal and transmembrane domains leading to aberrant localization of the fusion product, thus avoiding normal intracellular trafficking and degradation ${ }^{4}$. Currently, multi-kinase inhibitors with activity against RET are FDA approved for thyroid cancers, and clinical trials are investigating their use in targeting RET fusions in lung and other solid cancers $^{5,6}$.

Studies have demonstrated elevated expression of RET in ER+, HER2 + , and a subset of ER- breast cancers ${ }^{7,8}$. RET overexpression in $\mathrm{ER}+$ breast cancer was associated with resistance to tamoxifen and aromatase inhibitors ${ }^{9-11}$. In contrast, reduced RET expression, resulting from a $R E T$ polymorph, correlated with improved overall survival of patients with ER+ breast cancer ${ }^{12}$. Combining the aromatase inhibitor, letrozole, with a RET inhibitor has demonstrated improved efficacy over letrozole alone in preclinical models ${ }^{13}$ and clinical trials are evaluating the use of RET inhibitors to enhance sensitivity and reduce resistance to hormonal therapies in breast cancers (clinicaltrials.gov).

Although the role of RET expression in ER+ breast cancer has been under investigation, a comprehensive analysis of the presence and frequency of recurring RET genomic alterations, particularly $R E T$ rearrangements in breast cancer has not been reported. Unlike targeted or whole exome sequencing approaches, intron-capture-based approaches that involve baiting of targeted intronic regions can identify rearrangements, including translocations, inversions, tandem duplications or small deletions. By high depth sequencing of hotspot introns that precede the kinase domain-coding exons, breakpoints of rearrangements affecting these regions can be identified. Similar to RET mutations, RET rearrangements in breast cancer may serve as predictive biomarkers and be therapeutically actionable using small molecule kinase inhibitors ${ }^{14-16}$.

Using targeted genomic profiling with hybrid capture that includes analysis of introns 9, 10, and 11 of RET, high-level analysis of genomic alterations of RET in a variety of breast cancer subtypes are presented here. Based on initial detection in two index cases with RET fusions (RASGEF1A-RET and NCOA4$R E T), 9693$ breast cancers were evaluated for the presence of $R E T$ rearrangements as well as missense mutations and copy number changes. Novel rearrangements and previously reported fusions in $R E T$ were identified in this comprehensive cohort of advanced breast cancer patients. The presence of a spectrum of structural events and copy number alterations involving RET is demonstrated here, including co-events, functionality, and therapeutic actionability of rearrangements and amplifications using in vitro and in vivo models. Lastly, clinical benefit was observed in the index case harboring NCOA4-RET after receiving the RET inhibitor cabozantinib.

\section{Results}

Genomic profiling identifies recurrent RET alterations. The landscape of RET genomic alterations in 9693 breast cancer samples was assessed as part of hybrid capture-based next-generation sequencing of up to 405 cancer-related genes including select introns of up to 25 genes (gene panels, Supplementary Data 1). Samples were sequenced to a high uniform depth of coverage (median exon coverage, $637 \times$ ). Median patient age was 54 years (range, 20-88) (Table 1) and all samples were from female patients. Tissue for genomic profiling was obtained from the breast for 3859 patients (40\%) and from metastatic sites for 5834 patients $(60 \%)$.

RET genomic alterations were observed in $1.2 \%$ (121/9693) of breast cancer cases. With one case harboring two RET alterations (RET amplification and RET rearrangement), a total of 122 RET genomic alterations were identified including 16 rearrangements, 25 missense mutations, and 81 amplifications (median copy number $=8$, copy number range $=6-21$ ) (Fig. 1a, Table 1, Supplementary Data 2). ER status, based on immunohistochemistry, was available for $81 \%(98 / 121)$ of $R E T$ altered cases and HER2 (ERBB2 amplification) status, based on genomic profiling, was available for all cases (Fig. 1a, Table 1). RET genomic alterations were detected across all breast cancer subtypes although a majority were $\mathrm{ER}-(65 \%)$ or ERBB2 nonamplified (82\%). In comparison to cases with other types of RET genomic alterations (Fig. 1a, Table 1), the subset of cases with RET missense mutations was more frequently ER+ $(71 \%)(p=0.0002$, Fisher's exact test, two-tailed; Table 1$)$. RET rearrangements were more frequently ER- $(75 \%)(p=0.1615$, Fisher's exact test, twotailed; Table 1), as were RET amplifications (75\%) $(p=0.0077$, Fisher's exact test, two-tailed; Table 1). For the 121 RET altered cases, the most frequent altered genes that co-occurred with $R E T$ were TP53 (80\%), MYC (32\%), PIK3CA (26\%), ERBB2 (20\%), MCL1 (20\%), and PTEN (17\%) (Supplementary Table 1).

Rearrangements were identified by hybrid capture using probes for selected hotspot introns (introns 9-11) and all exons of RET. Based on detection of breakpoints, all rearrangements retaining intact sequences for RET kinase domain-coding exons (12-19), with or without $\mathrm{N}$-terminal fusion partner genes were included for the analysis. Out of the 16 RET rearrangements identified, 8 were defined as activating fusions, and the other 8 as uncharacterized rearrangements. Of the eight activating fusion events predicted to contain the kinase domain of RET, seven cases were either CCDC6-RET $(n=6)$ or NCOA4-RET $(n=1)$ that have been previously characterized as oncogenic ${ }^{17-19}$ and are recurrent in NSCLC ${ }^{20}$ and PTC (Fig. 1a, b); one case harbored a novel RASGEF1A-RET fusion subsequently characterized here as functional and activating.

Of the total eight unique uncharacterized rearrangements identified, novel gene partners, duplications, and truncations were observed (Fig. 1c). One case harbored an RET-RASGEF1A rearrangement. Although this rearrangement did not include the exons encoding the kinase domain, the similarity of the genomic breakpoints to the RASGEF1A-RET fusion (Fig. 1b) suggests the potential for a reciprocal rearrangement event that was not detected but could potentially lead to an activating fusion. One case harbored a ZNF485-RET rearrangement where ZNF485 is a putative novel fusion partner; four cases harbored full-length $R E T$ (including the $3^{\prime}$ untranslated region or UTR) followed by tandem duplication of the RET $3^{\prime}$ exons including exons 12-19 that encode the kinase domain; two cases harbored rearrangements with a breakpoint at RET intron 11 with exons encoding the kinase domain juxtaposed with intergenic space.

Missense mutations in RET were detected in 25 cases (Fig. 1d) and the majority were characterized as activating $(64 \%, 16 / 25)$. Observed recurrent, $R E T$ activating point mutations included the E511K extracellular domain mutation $(n=5)^{21}$ and V804M kinase domain mutation $(n=4)^{3}$; other activating mutations that were observed include extracellular domain mutation C611R, C620F, L633V, C634R, C634F, T636M, and the kinase domain $\mathrm{M} 18 \mathrm{~T}^{3,22}$. The remaining missense mutations have been previously described as somatic in cancer but have not been characterized $^{23}$. RET missense mutations are known to be causally associated with MEN2 syndrome (multiple endocrine neoplasia type 2). Using a computational method, missense 
a

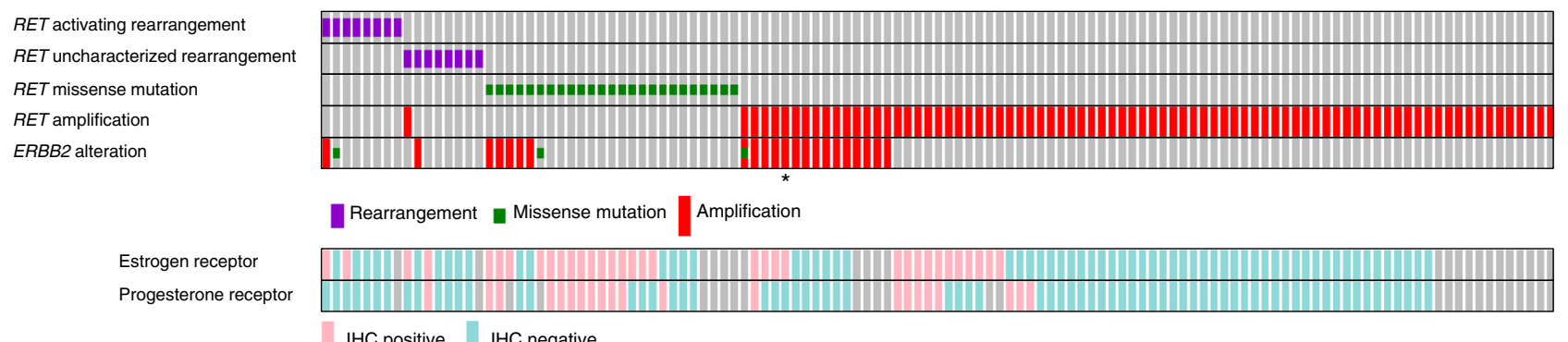

IHC positive IHC negative

b

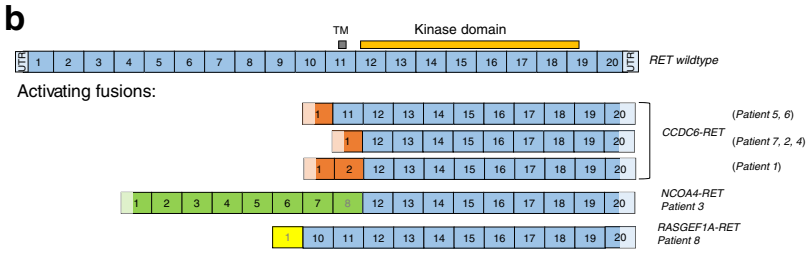

C

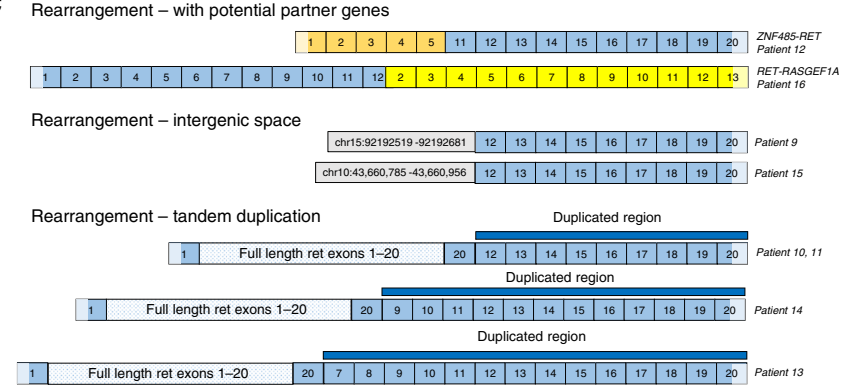

d

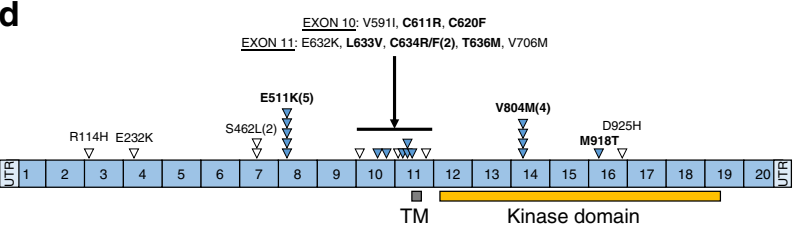

e

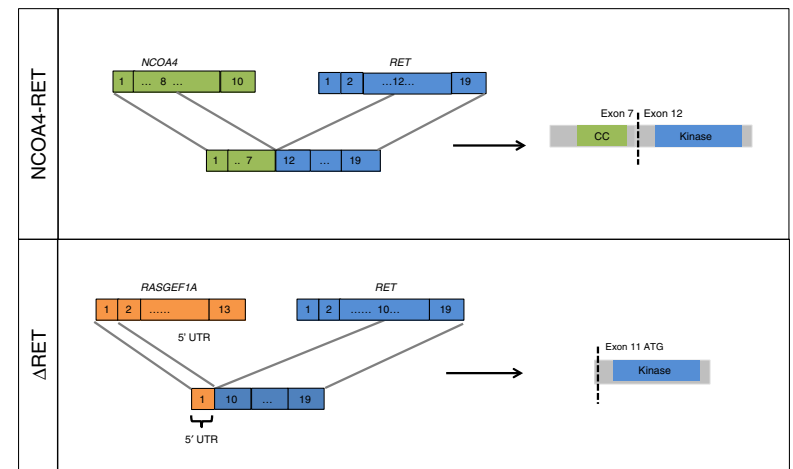

Fig. 1 Recurrent RET rearrangements in breast cancer. a OncoPrint of RET alterations in breast cancer. A case-by-case comparison of 121 breast cancers (column) carrying either a RET activating or uncharacterized rearrangement, missense mutation, or amplification (rows) to their ERBB2 amplification status (row) by clinical genomic profiling and available ER (Estrogen Receptor), PR (Progesterone Receptor) status (rows) from routine immunohistochemistry, generated using the oncoprint software ${ }^{58,59}$. Only one case harbored both a RET rearrangement and a RET amplification. ${ }^{\star} \mathrm{RET}$ protein expression in tissue from case with RET amplification is verified by western blot (Supplementary Fig. 4c). $\mathbf{b}$ RET fusions. Exon composition comparing full-length, wild-type RET with activating fusions identified in this study. Activating fusions maintain the exons (12-19) required for an intact kinase domain. Six patient samples carried the CCDC6-RET fusion. NCOA4-RET and a novel RASGEFTA-RET fusion initially detected in the index cases are functionally characterized in this report. UTR untranslated region, TM Transmembrane domain. c RET rearrangements. Exon composition of rearrangements including RET exons fused with novel partner genes (ZNF485, RASGEFTA), rearrangements of kinase domain-coding exons 12-19 of RET into intergenic space, rearrangements resulting in tandem duplications that involve exons 12-19 of RET. d RET point mutations. Schematic depicting location and number of the 25 RET mutations. Filled triangles and bold font indicate characterized activating mutations based on literature and open triangles represent uncharacterized mutations that have been described as somatic in cancer. Number in bracket represents number of cases. e lllustration of index case fusions (NCOA4-RET and RASGEFTA-RET) depicting breakpoints in exon 8 for NCOA4 and intron 11 for RET resulting in a product encoding NCOA4 (exons 2-7) fused to RET (exons 12-19). Breakpoints in intron 1 of RASGEFTA and intron 9 of RET modeled to result in an N-terminally truncated product $\triangle$ RET, as exon 1 of RASGEFTA is part of $5^{\prime}$ UTR and exon 11 of RET contains a potential alternate start site. CC coiled-coil domain, UTR untranslated region, ATG methionine start codon

mutations were analyzed to determine germline versus somatic variant status based on allele frequencies, altered copy number, and tumor purity and further verified using a second algorithm in cases where ploidy was two (Supplementary Table 2) ${ }^{24,25}$. Out of 25 missense mutations, 12 were germline and 7 were somatic. The remaining six were categorized as ambiguous and require further analysis using other approaches.

Two fusions were further analyzed in detail. The NCOA4-RET fusion was detected in an ER $+/ \mathrm{PR}-/ \mathrm{HER} 2+$ breast cancer and results from tandem duplication with breakpoints in NCOA4 exon 8 and RET intron 11; includes the NCOA4 exons encoding a putative coiled-coil domain and the RET exons encoding the kinase domain and therefore retains all the functional domains characteristic of activating NCOA4-RET fusion proteins that have been studied in PTC and NSCLC $3,17,20,26$ (Fig. 1e). The novel RASGEF1A-RET fusion was detected in an ER-/PR-/HER2breast cancer and results from an inversion event on chromosome 10 with breakpoints in RASGEF1A intron 1 and RET intron 9 that juxtaposes the 5'UTR of RASGEF1A upstream of the RET kinase domain.

To characterize the novel RASGEF1A-RET fusion, exons 10-19 of RET were analyzed for the presence of an alternate internal start site. A methionine codon in exon 11 was identified that could potentially translate an $\mathrm{N}$-terminally truncated RET protein with an intact RET kinase domain and this variant was designated as $\triangle R E T$. RASGEF1A does not contribute to the amino-acid sequence of $\triangle \mathrm{RET}$ as it only encompasses the RASGEF1A 5'UTR. $\triangle R E T$ may also serve to model some of the 


\section{Table 1 Clinicopathologic characteristics of RET altered breast cancers}

\begin{tabular}{|c|c|c|c|c|c|c|}
\hline Characteristics & $\begin{array}{l}\text { All breast } \\
\text { cancer cases }\end{array}$ & $\begin{array}{l}\text { RET } \\
\text { altered } \\
\text { cases }\end{array}$ & $\begin{array}{l}\text { RET activating } \\
\text { rearrangement }\end{array}$ & $\begin{array}{l}R E T \text { uncharacterized } \\
\text { rearrangement }\end{array}$ & $\begin{array}{l}\text { RET missense } \\
\text { mutation }\end{array}$ & $\begin{array}{l}\text { RET } \\
\text { amplification }\end{array}$ \\
\hline Median age (range), years & $54(20-88)$ & $56(31-85)$ & $61.5(54-66)$ & $60(48-69)$ & $52(33-71)$ & $54(28-85)$ \\
\hline $\begin{array}{l}\text { Median tumor mutational burden } \\
\text { (range), mutations } / \mathrm{Mb}\end{array}$ & $3.6(0-251.4)$ & $\begin{array}{l}4.5 \\
(0-36.6)\end{array}$ & $5.2(0.9-7.2)$ & $4.5(1.8-17.1)$ & $3.6(0-36.6)$ & $4.5(0-16.2)$ \\
\hline $\begin{array}{l}\text { Breast }(n) \text { as site of origin of } \\
\text { sequenced sample }\end{array}$ & 3859 & 49 & 4 & 4 & 7 & 34 \\
\hline ER positive & NA & $34(34.7 \%)$ & $2(25 \%)$ & $2(25 \%)$ & $15(71.4 \%)$ & $16(25 \%)$ \\
\hline ER negative & NA & $64(65.3 \%)$ & $5(75 \%)$ & $5(75 \%)$ & $6(28.6 \%)$ & $48(75 \%)$ \\
\hline ER unknown & 8221 & 23 & 1 & 1 & 4 & 17 \\
\hline ERBB2 amplified & $1019(10.5 \%)$ & $22(18.2 \%)$ & $1(12.5 \%)$ & $1(12.5 \%)$ & $5(20.0 \%)$ & $15(18.5 \%)$ \\
\hline ERBB2 nonamplified & $8674(89.5 \%)$ & $99(81.8 \%)$ & $7(87.5 \%)$ & $7(87.5 \%)$ & 20 (80.0\%) & $66(81.5 \%)$ \\
\hline
\end{tabular}

intergenic RET rearrangements (Fig. 1c) which are missing exons $1-10$ but preserve kinase domain-coding exons $12-19$. If using an internal methionine codon in exon 12, the intergenic rearrangements would also be N-terminal truncation mutants, similar to $\triangle R E T$, but missing more $\mathrm{N}$-terminal residues from exon 11 and some kinase domain residues from exon 12. Since they are intergenic rearrangements, unlike RASGEF1A-RET, they could also potentially use alternate transcription initiation mapping prior to RET exons and, if in-frame, may include an intact RET kinase domain.

Breast cancer RET fusions are constitutively active. To assess the functionality of NCOA4-RET and RASGEF1A-RET, fusion constructs for NCOA4-RET and open reading frame (ORF) for $\triangle R E T$ (product of RASGEF1A-RET) were developed and expressed in immortalized mouse fibroblasts NIH/3T3 and nontumorigenic MCF10A human epithelial mammary cells. Fulllength, wild-type RET was ectopically expressed in order to model $R E T$ amplification leading to overexpression and is hereby referred to as RET ${ }^{\mathrm{amp}}$. Western blot analysis using an antibody against C-terminus of RET detected NCOA4-RET and $\triangle$ RET bands at predicted sizes of 68 and $46 \mathrm{kDa}$ in the transduced cells respectively (Fig. 2a). To assess the oncogenic potential of the fusions, nontumorigenic NIH/3T3 and MCF10A cells expressing empty vector, RET ${ }^{\mathrm{amp}}, \triangle \mathrm{RET}$, or NCOA4-RET were evaluated for cell growth and colony formation. Expression of both fusions and RET ${ }^{\mathrm{amp}}$ resulted in a significantly increased growth capacity (Fig. 2b and Supplementary Fig. 1a). Increased clonogenic expansion was observed in NIH/3T3 cells when compared to vector control (Fig. 2c). In the absence of growth factor stimulation, expression of NCOA4-RET, $\triangle$ RET, and RETamp resulted in phosphorylation at tyrosine 905 (kinase activation loop tyrosine of RET) and tyrosine 1062 (major signaling hub of RET kinase to MAPK and PI3K-AKT pathways) consistent with constitutive kinase activation (Fig. $2 \mathrm{~d}$ and Supplementary Fig. 1b). The kinase inactive mutant, K758M-RET, was used as a negative control and the constitutively active kinase mutant, M918T-RET, was used as a positive control. Increased signaling in the presence of RET alterations was confirmed for either RASMAPK pathway by phosphorylation of MEK or PI3K-AKT pathway by downstream phosphorylation of P70 S6 kinase (Fig. 2e).
Breast cancer RET fusions confer sensitivity to RET inhibition. RET fusions were evaluated for sensitivity to FDA-approved kinase inhibitors known to have activity against RET (Fig. 3 and Supplementary Fig. 2). Cabozantinib and sorafenib effectively reduced viability of $\mathrm{NIH} / 3 \mathrm{~T} 3$ and MCF10A breast cell lines expressing both RET fusions in an antiproliferative MTS assay (Fig. 3a and Supplementary Fig. 2a). In order to account for differences in growth rate, cell numbers were plated differentially for NIH/3T3 cells to ensure $80 \%$ confluence in vehicle-treated wells on the day of viability measurement. In the case of MCF10A, equal numbers were plated for all cell lines since growth differences were not observed between the cell lines at day 4 of the MCF10A growth assay, which coincides with the time duration from plating to reading of the MTS assay and does not confound the drug response results observed (Supplementary Fig. 1a). Further evaluation with cabozantinib in RET fusion cell lines by incubating with increasing concentrations of the drug revealed a dose-dependent reduction in phosphorylation of RET fusion kinase and downstream signals MEK and P70 S6 by western blot (Fig. 3b, Supplementary Fig. 2b) suggesting that the effect was driven by RET inhibition. Further, inhibition of downstream signaling was enhanced in NCOA4-RET cells in comparison to vector cells, which are negative for RET expression by western blot, thus suggesting the effect was mainly driven by targeting the RET fusion (Supplementary Fig. 3a). In addition, cabozantinib and another RET inhibitor, vandetanib, were able to effectively inhibit the enhanced colony-forming abilities of RET fusion cell lines and resulted in a significant reduction in size and number of colonies in a dose-dependent fashion in comparison to no-drug treatment and control cell lines (Fig. 3c, Supplementary Fig. 3b).

RET fusions are tumorigenic. NIH/3T3 cells transduced with RET fusions formed tumors in athymic nude mice within 2 weeks of subcutaneous injections (1 million cells, bilateral flank injections) for NCOA4-RET and 5 weeks for $\triangle$ RET, whereas vector and RET $^{\text {amp }}$ cells did not form tumors when examined for 10 weeks (Fig. 4a). To further explore tumor formation for RET $^{\text {amp }}$ cells, higher numbers (4.5 million cells, bilateral flank) were injected into NOD/SCID/interleukin 2 receptor $\gamma$ null mice, which is a more powerful immunodeficient model compared to athymic nude mice. RET ${ }^{\mathrm{amp}}$ mice formed tumors within 7 weeks 
a

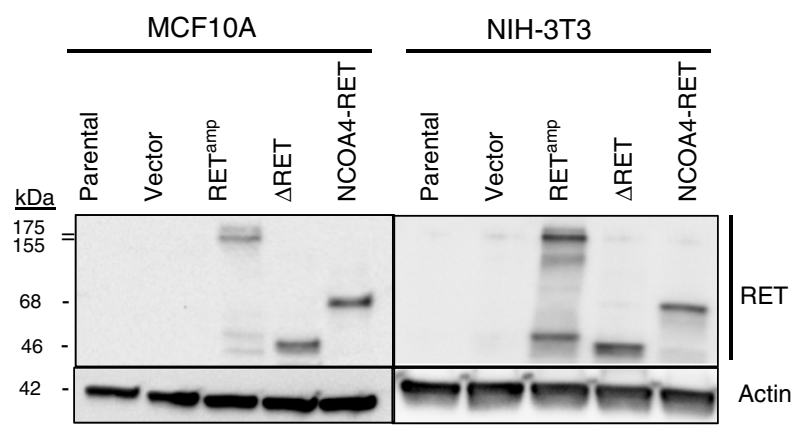

b

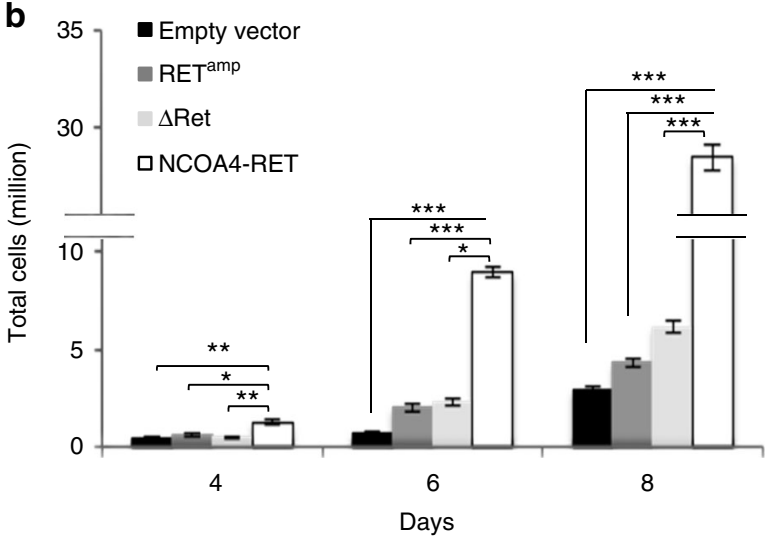

C

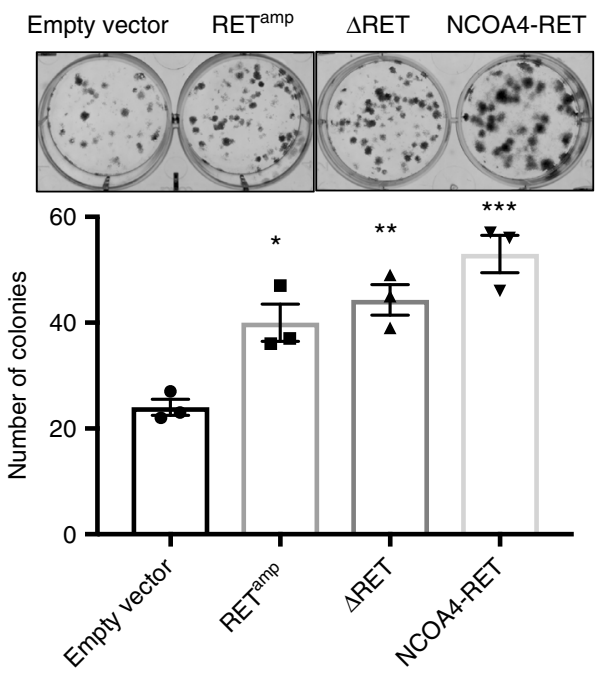

d

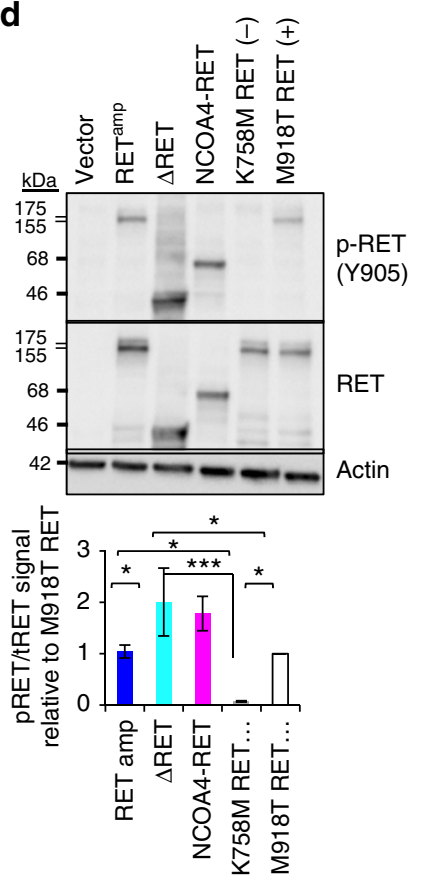

e

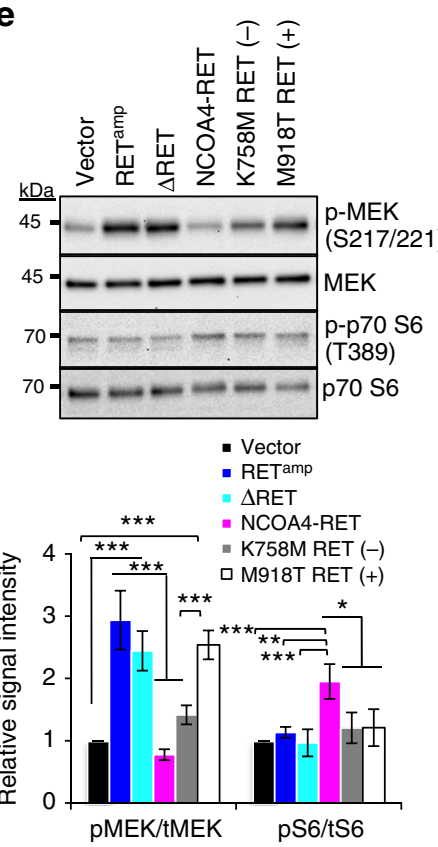

Fig. 2 Transforming activity and oncogenic signaling of RET alterations. a MCF10A (human breast epithelial) and NIH/3T3 (immortalized mouse fibroblasts) cells overexpressing RET wild-type (RET ${ }^{\mathrm{amp}}$ ), NCOA4-RET, and $\triangle$ RET. Expressed proteins were detected using a C-terminal RET antibody at predicted sizes of $155 / 175,68$, and 46 kDa respectively. NIH/3T3 cells transduced with RETamp, NCOA4-RET, and $\triangle$ RET show increased $\mathbf{b}$ growth rates and $\mathbf{c}$ clonal expansion compared to cells transduced with vector alone. For growth curve experiments, 20,000 cells were plated per dish in triplicate for all cell lines and counted at days 4, 6, and 8. For clonogenic studies, 150 cells were plated per well in triplicate for all cell lines and stained with crystal violet at the end of 14 days. $\mathbf{d}$ Immunoblot analysis of NIH/3T3 cells overexpressing RETamp, $\triangle$ RET, and NCOA4-RET reveal phosphorylation at tyrosine 905 and e downstream signaling measured after serum starvation for $24 \mathrm{~h}$. In (d) and (e), kinase inactive mutant (K758M) and constitutively active mutant (M918T) refer to full-length RET variants used as negative and positive controls respectively. Results shown are representative of experiments performed thrice and error bars indicate s.d. $(n=3) . p \leq 0.05\left(^{\star}\right), \leq 0.01\left(^{\star \star}\right), \leq 0.001\left(^{\star \star \star}\right)$ are statistically significant and analyzed by ANOVA and Tukey's multiple comparisons test. Open-ended brackets depict comparison between the indicated group(s) and each of the groups under the bracket. Where brackets are absent, comparison is with empty vector

whereas matched vector cells did not form tumors when examined for 10 weeks (Fig. 4b). Together, the data in Fig. 4a, b suggest that while RET fusions are tumorigenic, constitutive activation of the RET kinase by RET overexpression alone (RET ${ }^{\mathrm{amp}}$ ) is also capable of driving tumor growth under sufficiently permissive conditions. Immunohistochemistry revealed positive staining for the proliferation marker Ki-67 for RET ${ }^{\mathrm{amp}}, \mathrm{BRET}$, and NCOA4RET tumor tissues (Fig. 4c). Tumor protein was verified for the expression of RET fusions by immunoblot and activation of PI3K-AKT and MAPK pathways was observed (Fig. 4d). Based on experiments described in NIH/3T3 cells (Figs. 2b and 4a), NCOA4-RET was observed to be the fastest growing cell line and this translated to tumor formation in less than 2 weeks; whereas $\triangle \mathrm{RET}$ and $\mathrm{RET}^{\mathrm{amp}}$ were slower in comparison, taking longer than a month. Next, the effect of RET inhibitor, cabozantinib on tumor response was tested in each xenograft model. In order to offset the time for tumor formation, $0.5 \times 10^{6}$ cells were injected for NCOA4-RET, whereas $5 \times 10^{6}$ cells were injected for $\triangle \mathrm{RET}$ and RET $^{\mathrm{amp}}$ in athymic nude mice.

RET-fusion-driven tumors respond to a RET inhibitor. Cabozantinib was used in xenograft models for NCOA4-RET, $\triangle$ RET, and RET ${ }^{\text {amp }}$ to determine drug sensitivity in vivo. In each case, 

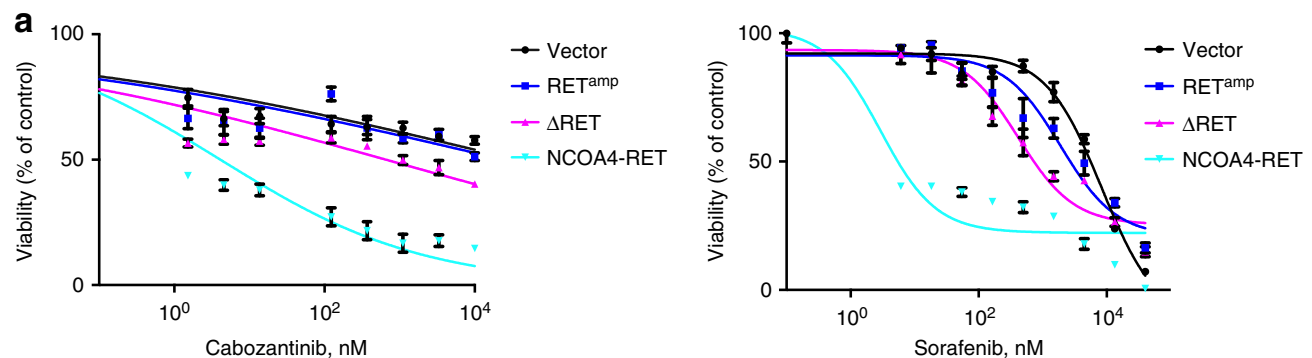

b

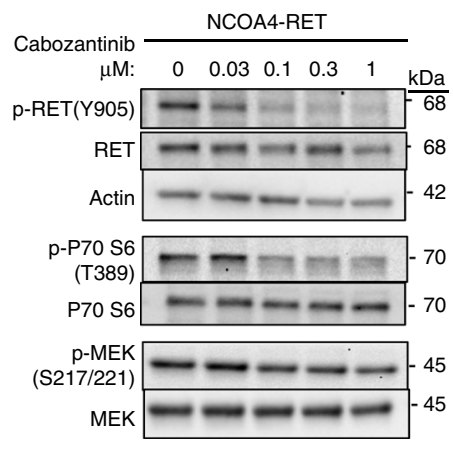

- $0 \quad=30 \quad=100 \quad-300 \quad \square \quad 1000$ Cabozantinib, nM

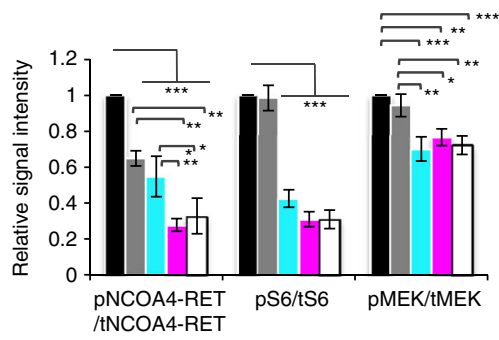

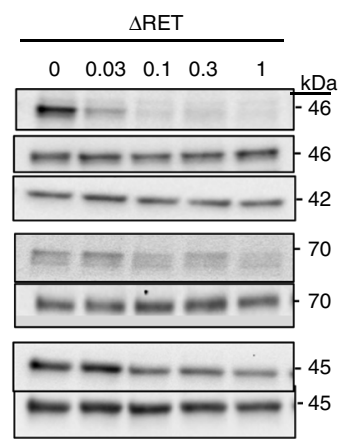

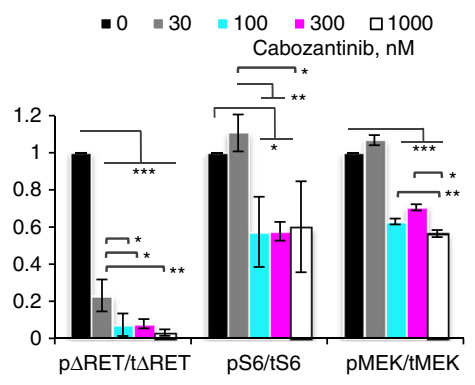

C
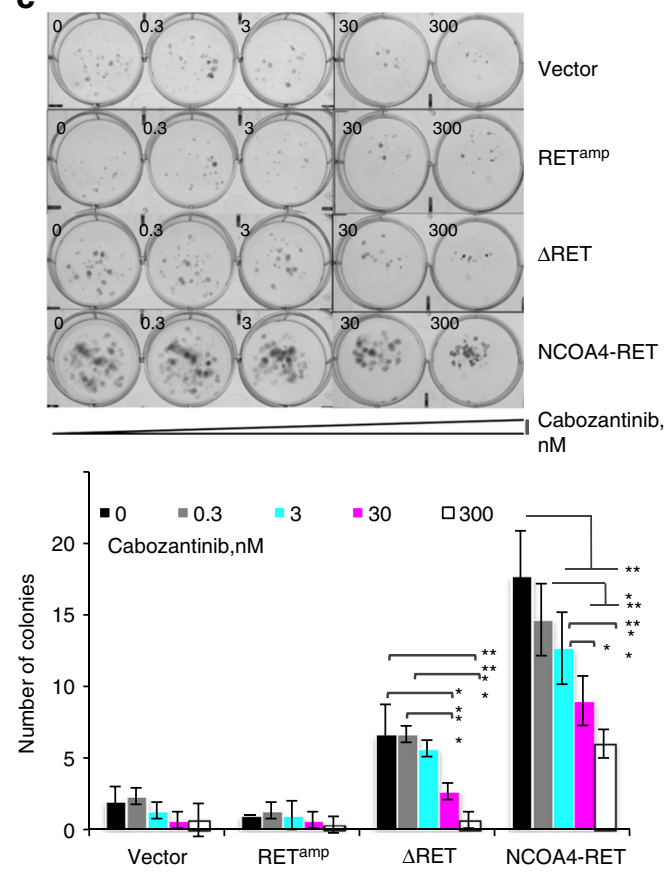

Fig. 3 Fusion cell lines exhibit dose-dependent response to RET inhibition. a Dose-response curves after $72 \mathrm{~h}$ of drug treatment with cabozantinib or sorafenib in NIH/3T3 cells expressing RETamp, $\triangle$ RET, NCOA4-RET, and vector. Cell viability normalized to vehicle (DMSO)-treated cells. Error bars indicate s.d. of three replicates and are representative of three independent experiments $(n=3)$. b Western blot indicating inhibition of RET fusion kinase, MEK and P70 S6 signaling with increasing concentration of cabozantinib in NIH/3T3 cells transiently expressing NCOA4-RET or $\Delta$ RET. Measurements were made after overnight serum starvation and $1 \mathrm{~h}$ of incubation with cabozantinib in the absence of serum. Graphs represent image densitometry analysis of western blots from three independent experiments $(n=3)$. Ratio of phosphorylated to total proteins is measured at each concentration and mean values with error bars indicating s.d. are plotted relative to DMSO-treated control. c Dose-dependent reduction in $\triangle$ RET and NCOA4-RET colony numbers upon treatment with increasing concentrations of cabozantinib in NIH/3T3 transduced cells for 14 days. 0 represents DMSO-treated controls. All cells were plated at equal numbers per well in triplicates per experiment. Error bars indicate s.d. of three replicate measurements per condition ( $n=3$ ) and are representative of experiments performed three times. $p \leq 0.05\left({ }^{\star}\right), \leq 0.01\left(^{* \star}\right)$ and $\leq 0.001$ (***) by ANOVA (one-way for (b) and two-way for (c)) with Tukey's multiple comparisons test. Open-ended brackets depict comparison between the indicated group and each of the groups under the bracket. Where brackets are absent, comparison is with DMSO control

upon tumor formation, mice were randomized into three groups and treated with either a low dose $\left(30 \mathrm{mg} \mathrm{kg}^{-1}\right)$, high dose $(60$ $\mathrm{mg} \mathrm{kg}^{-1}$ ) of cabozantinib, or vehicle saline. Treatment with 60 and $30 \mathrm{mg} \mathrm{kg}^{-1}$ effectively inhibited tumor growth for NCOA4RET xenografts and resulted in rapid regression of the tumor within 2 weeks (Fig. 5a). In case of $\triangle$ RET, both doses inhibited tumor growth and a dose-dependent effect was observed, with the higher dose of $60 \mathrm{mg} \mathrm{kg}^{-1}$ resulting in a more significant reduction in tumor volume when compared to $30 \mathrm{mg} \mathrm{kg}^{-1}$ at the end of 2 weeks (Fig. 5b). RET amp xenografts also showed significant reduction in tumor volumes with both doses of cabozantinib in comparison to vehicle, despite increased variability between mice from the same treatment group (Supplementary Fig. $4 \mathrm{a}$ and $4 \mathrm{~b}$ ). Overall, results indicated that all three RET alterations are sensitive to cabozantinib as evidenced by significant tumor volume reduction.
Tumor protein, collected at the end of treatment on day 14 for NCOA4-RET and $\triangle$ RET, revealed significant reduction in the fusion protein levels and downstream PI3K-AKT signaling for the cabozantinib-treated groups as measured by western blot (Fig. 5c and Supplementary Fig. 4d). Histological analysis of the xenograft tissue for NCOA4-RET and $\triangle$ RET shows a stark contrast in tumor cellularity between vehicle and treatment groups (Fig. 5d), supporting the reduction of fusion proteins observed in Fig. 5c. A packed population of highly proliferative cells as revealed by Ki67 stain in vehicle tissue is efficiently cleared in the treatment groups in a dose-dependent manner. The treated tumors reveal hyalinization and apoptosis. Treated tumor tissues stained positive for Cleaved Caspase-3 whereas vehicle tissue was relatively negative verifying activation of the apoptotic pathway for tumors treated with cabozantinib (Fig. 5d). Xenograft data with RET $^{\mathrm{amp}}$ are suggestive of a benefit; however, the large 
a
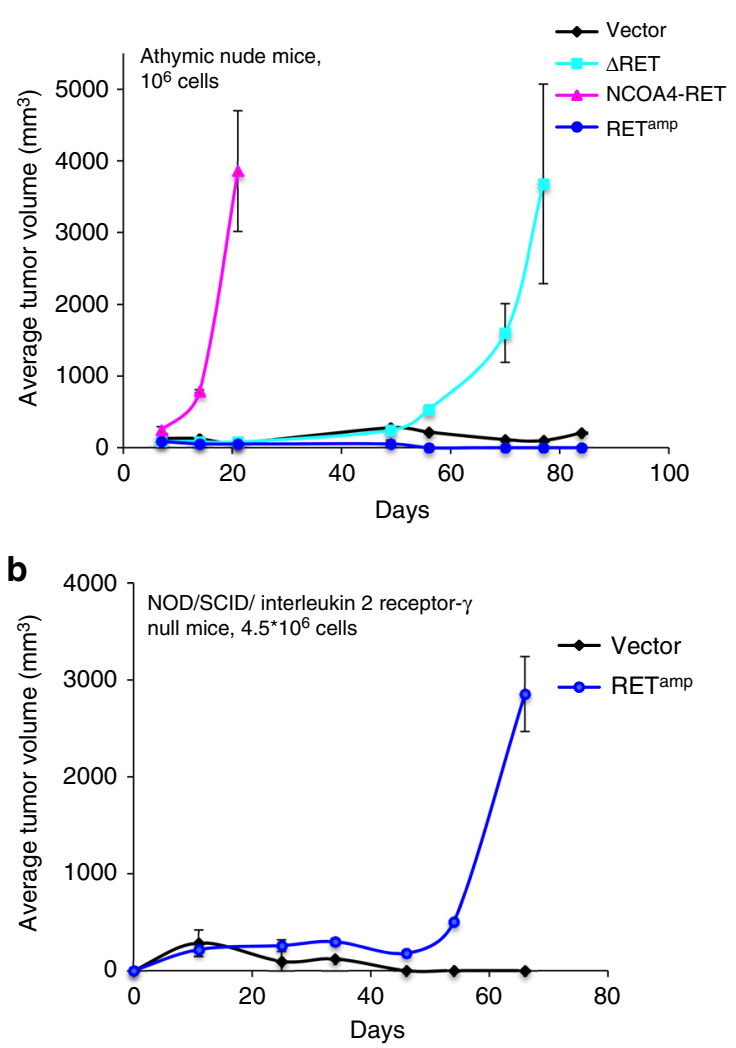

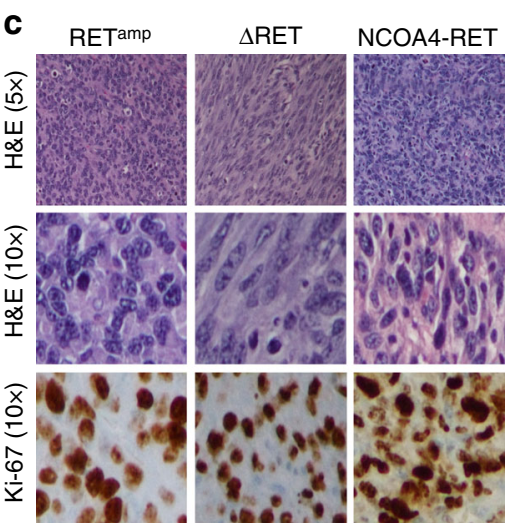

d

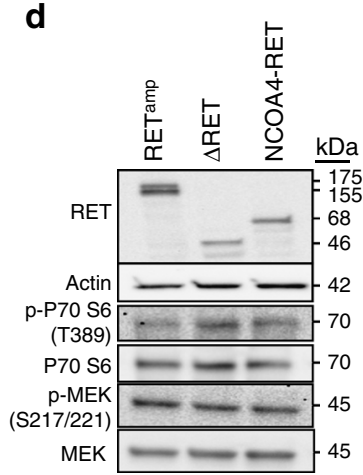

Fig. $4 \Delta$ RET and NCOA4-RET are tumorigenic. Growth curve of tumors formed upon subcutaneous injection of transduced NIH/3T3 cells at a $1 \times 10^{6}$ cells, bilateral flank injections in athymic, nude mice $(n=4$ injection sites for vector, $\Delta$ RET, and RETamp and $n=6$ injection sites for NCOA4-RET) and $\mathbf{b}$ $>4.5 \times 10^{6}$ cells, bilateral flank injections in NOD/SCID/ interleukin 2 receptor $\gamma$ null mice $(n=4$ injection sites per group) for vector and RETamp cells. Error bars represent mean \pm s.d. c Representative staining for hematoxylin and eosin (H\&E) demonstrates a packed population of tumor cells (top row, $\times 5$ magnification). At higher power (middle row, $\times 10$ magnification) tumor cells reveal mitoses. Tumor cells stain positive after immunohistochemistry for proliferation marker Ki-67 (bottom row, $\times 10$ magnification). d Immunoblot of tumor protein lysates from NIH/3T3 xenografts using the C-terminal RET antibody and downstream signaling proteins

confidence intervals and smaller differences compared to control should be interpreted more cautiously and warrants further exploration (Supplementary Fig. $4 \mathrm{a}$ and $4 \mathrm{~b}$ ).

NCOA4-RET positive breast cancer responds to cabozantinib. A 63-year-old female with a remote history of a stage I, ERbreast cancer (24 years prior) and a more recent stage I, ER +/HER2 - breast cancer (13 years prior, Fig. 6a) was found to have stage IV disease in 2014 . The patient initially had a lumpectomy followed by adjuvant radiation only, but required a mastectomy with axillary lymph node dissection for the second occurrence which was in the same breast followed by four cycles of doxorubicin and cyclophosphamide and then by 5 years of tamoxifen. In 2014, a regional/distant recurrence of ER+/HER2+ (by immunohistochemistry) disease (Fig. 6b) was confirmed on ultrasound-guided biopsy of the right axillary tail and both MRI and PET/CT imaging showing bony metastasis. Biopsy tissue was sent for genomic profiling (FoundationOne, Foundation Medicine) that identified the NCOA4-RET fusion and, based on the breakpoints, was found to join exons $1-7$ of NCOA4 in frame with exons $12-19$ of RET. Histology of the recurrent breast cancer tissue revealed a papillary architecture which is characteristic of RET fusion-positive thyroid cancers. Palliative radiation to the thoracic-lumbar spine followed by HER2targeted treatment with pertuzumab, trastuzumab, and anastrazole was initiated. Subsequently, axillary and bony progression was found and second-line therapy with combined trastuzumab, exemestane, and cabozantinib was initiated. Cabozantinib was added as a genomic-guided targeted therapy based on the NCOA4-RET fusion observed in the recurrent tumor. In preclinical studies, NCOA4-RET fusion-driven models have previously been targeted with RET inhibitors ${ }^{27}$ and clinical studies have demonstrated responses to RET inhibitors for patients with RET fusions in NSCLC ${ }^{28-30}$, which further supported treatment for this patient. The patient was treated initially with $140 \mathrm{mg} \mathrm{day}^{-1}$ of cabozantinib but due to side effects such as shortness of breath, intermittent treatment and dose reduction to $100 \mathrm{mg} \mathrm{day}^{-1}$ was required, and ultimately, treatment had to be discontinued (Fig. 6c). For a total of 67 days on cabozantinib, which amounts to $80 \%$ of duration for second-line treatment, there was a rapid radiographic and clinical response along with improvement in dyspnea and epistaxis. Representative PET/CT images of the thoracic spine lesion (Fig. 6d), which was present prior to cabozantinib, showed a significant reduction in PET avidity after treatment. Whether the response was due solely to cabozantinib, a change in hormone treatment, or the combination of these therapies cannot be determined with certainty. However, addition of a RET inhibitor to treatment of an ER+/HER2 treatment-refractory, NCOA4-RET- positive breast cancer resulted in a clinical response. 
a
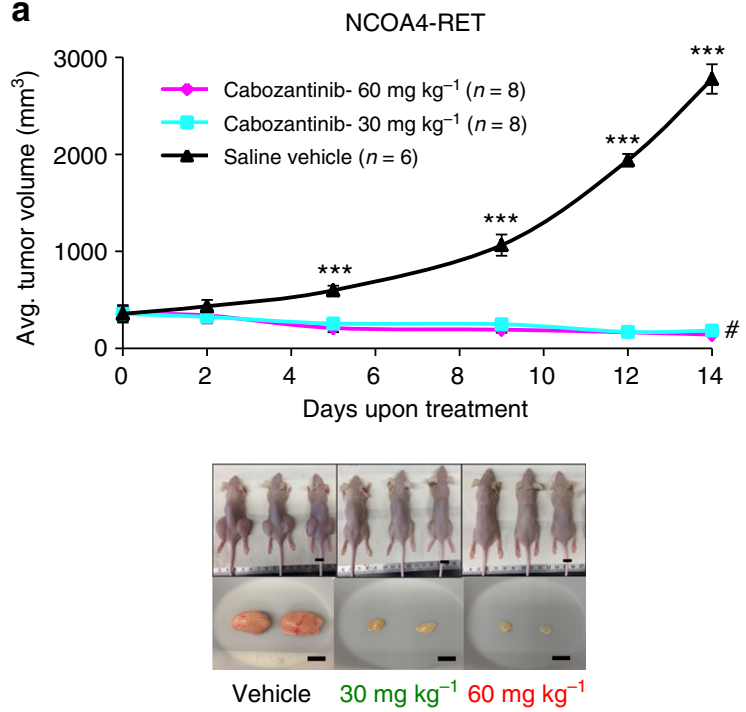

C
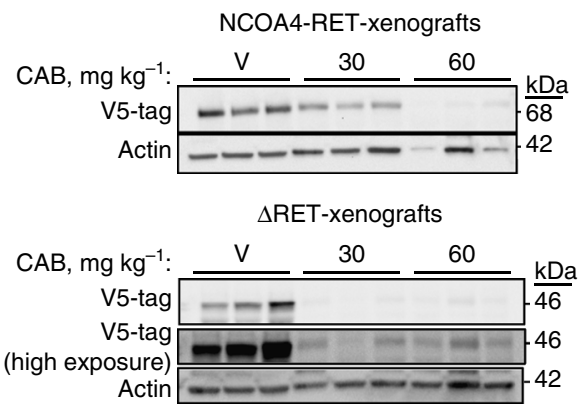

b
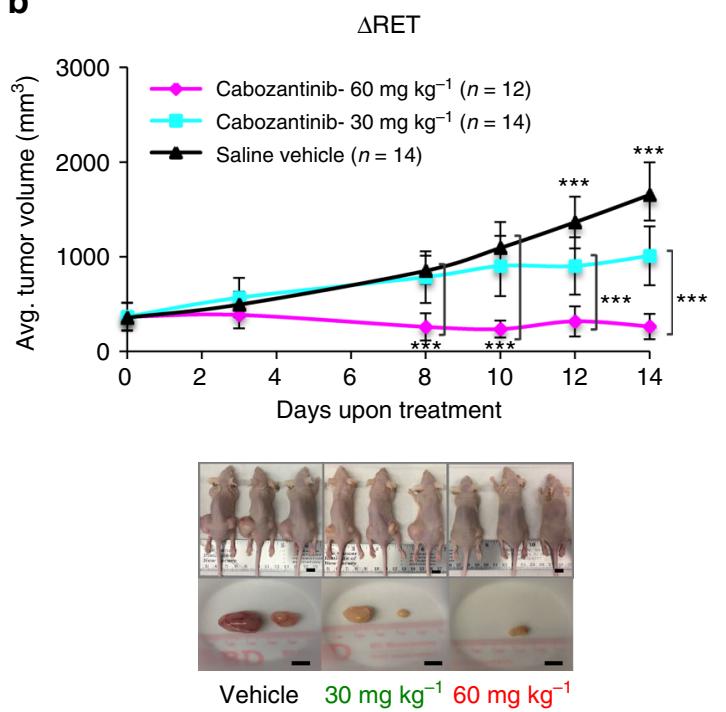

d

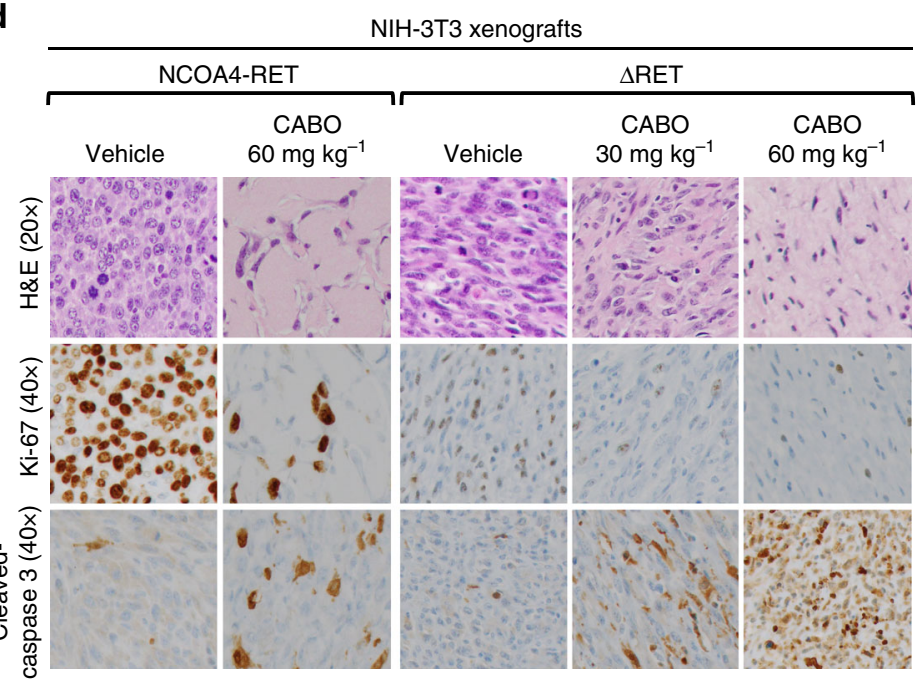

Fig. 5 Cabozantinib inhibits tumor growth driven by RET fusions. Mean tumor volume was measured in NIH/3T3 xenograft tumors driven by a NCOA4RET ( $n=8$ or 6 injection sites per group) or $\mathbf{b} \Delta \operatorname{RET}\left(n=14\right.$ or 12 injection sites per group) under treatment with either cabozantinib at $30 \mathrm{mg} \mathrm{kg}^{-1}, 60 \mathrm{mg}$ $\mathrm{kg}^{-1}$, or saline vehicle control for 14 days. Treatment started at day 0 . Error bars represent mean \pm s.d., ${ }^{\star \star \star}(p \leq 0.001)$ represent comparisons between both treatment groups and vehicle-treated controls when depicted above the vehicle curve or between indicated groups and \#( $p \leq 0.001)$ represents comparisons between day 0 and day 14 for the treatment groups (two-way ANOVA with Tukey's multiple comparison test). Mouse and tumor images are representatives from each treatment group at the end of study after 14 days of treatment. Scale bars indicate 10 mm. c Immunoblot of tumor protein lysates collected at the end of 14 days of treatment to measure changes in NCOA4-RET, $\triangle$ RET (detected by V-5 tag antibody, actin as loading control). Mice were treated on the day of tumor harvest for $4 \mathrm{~h}$ with saline vehicle or cabozantinib ( $30 \mathrm{or} 60 \mathrm{mg} \mathrm{kg}^{-1}$ ) and sacrificed. $n=3$ xenograft samples per treatment condition. $\mathbf{d}$ Representative hematoxylin and eosin (H\&E) staining of tumor tissue revealing a high grade sarcomatoid distribution (top row, $\times 20$ magnification) and immunohistochemistry of markers Ki-67, Cleaved Caspase-3 for comparison between vehicle and cabozantinib treatment groups in NCOA4-RET and $\triangle$ RET xenograft experiments (middle and bottom row, $\times 40$ magnification)

\section{Discussion}

In this study, RET alterations are reported from a large cohort of 9693 breast cancers that were genomically profiled as part of routine clinical care. Analysis of the RET genomic landscape has not been previously reported at this scale exclusively for breast cancer. Large sample size, high sequencing depth $(>600 \times)$, and hybrid capture to identify select introns in RET ensured accurate detection of all classes of genomic variants, robust statistical analyses as well as validation of rare variants resulting in a hit rate of $1.2 \%$. While this may be a relatively small fraction of patients, given that roughly 250,000 new cases of invasive breast cancer are diagnosed in the U.S. annually ${ }^{31}$, this extrapolates to roughly 3000 new cases each year in which RET alterations may become potentially relevant therapeutic targets. This is similar to the frequency of $A L K$ fusion-positive NSCLC diagnosed in the U.S. annually ${ }^{32}$. The fraction of advanced disease in this cohort is likely underestimated (Table 1) as these assays are often performed on breast tumor tissue when distant site tumor tissue is insufficient in quality or quantity. Considering the advanced or refractory nature of cases that undergo genomic profiling, 
a

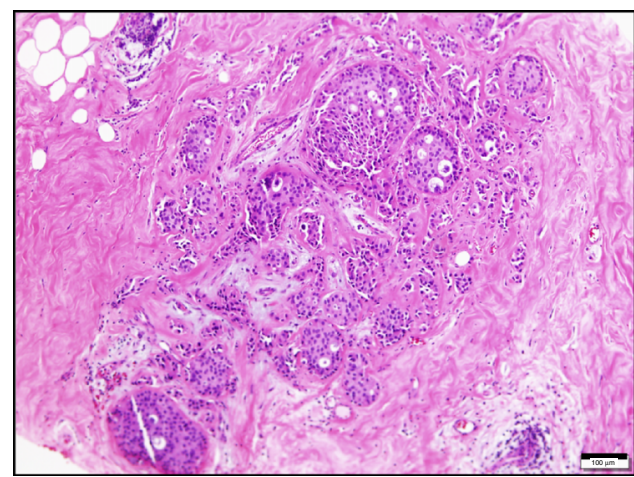

b

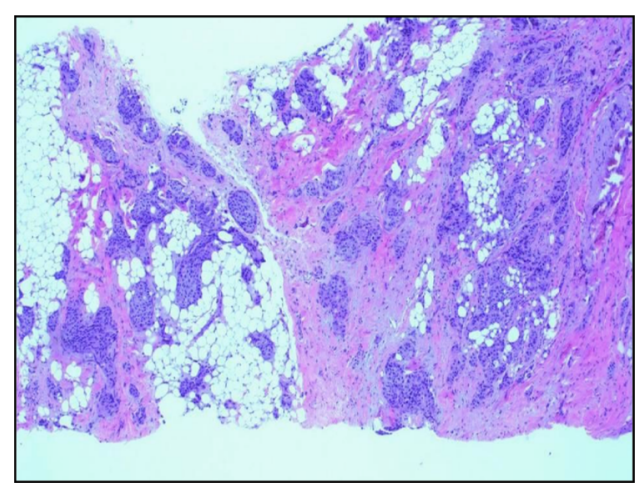

C Cabozantinib dose: 140 mgday $^{-1}$ 100 mgday $^{-1}$ 100 mgday $^{-1}$

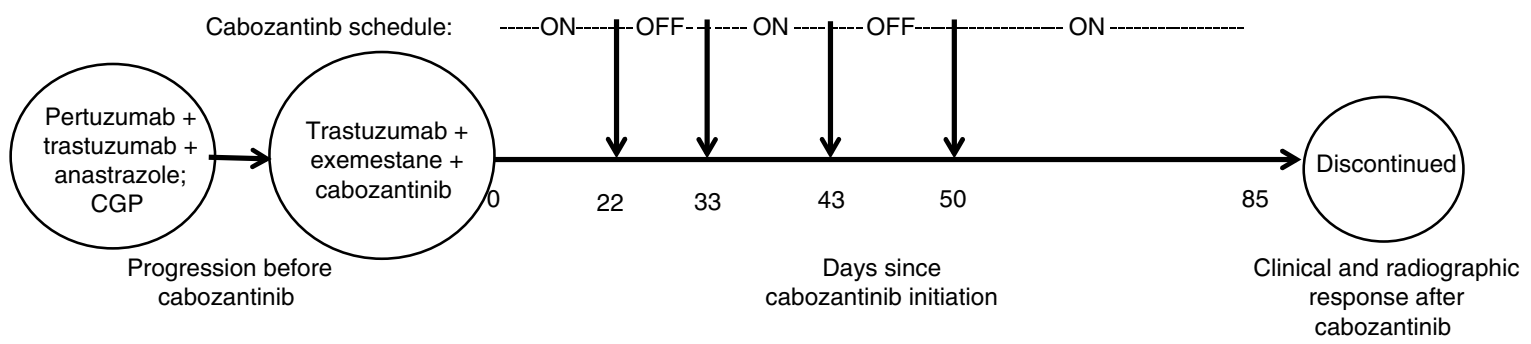

d

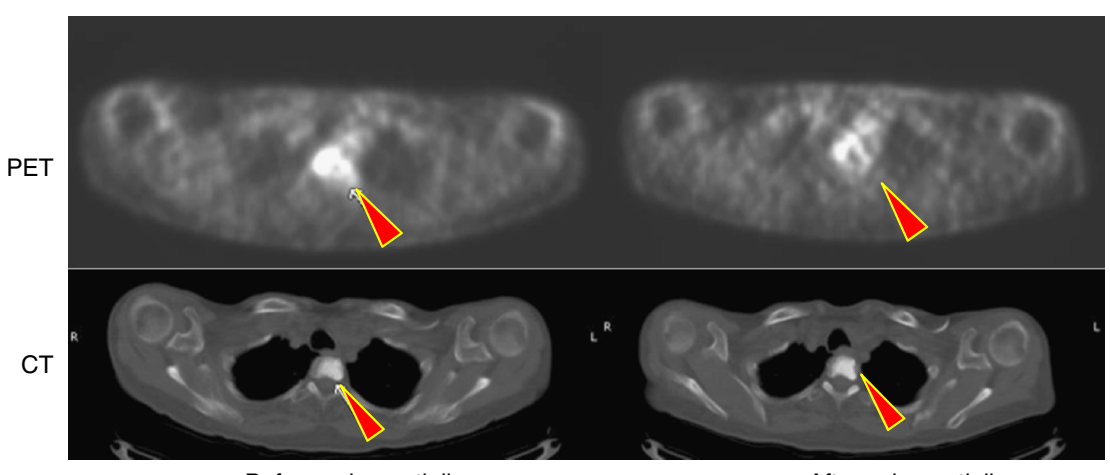

Before cabozantinib

After cabozantinib

Fig. 6 Clinical response to targeted therapy in a patient with RET-fusion+ve breast cancer. a Histology image of stage I ER+/HER2- tumor. Scale bar indicates $100 \mu \mathrm{m}$. b Histology of the regional/distant recurrence of ER+/PR-/HER2 3+ tumor from the right axillary tail, x4 magnification. Genomic profiling on this tissue revealed NCOA4-RET fusion (same as patient 3 in Fig. 1b). c Treatment schematic and timeline showing initiation of cabozantinib (day 0 ) after progressing on HER2-targeted treatment (pertuzumab, trastuzumab) and anastrazole. Targeted genomic profiling identified the presence of NCOA4-RET fusion. Based on the finding, cabozantinib, a RET inhibitor, was added along with trastuzumab and exemestane as second-line treatment. Intermittent treatment and dose reduction was required due to side effects. Clinical and radiographic response was observed 85 days after cabozantinib initiation. $\mathbf{d}$ Representative PET (top row) and CT (bottom row) images of thoracic spine lesion (filled red arrows) before and after cabozantinib treatment. PET signal avidity is reduced after treatment

identifying a targetable alteration holds promise for these patients who may face diminishing options using standard approaches.

Resistance to cancer treatment can arise from tumor evolution resulting in development of fusions, mutations, or amplifications and alternative, by-pass signaling pathways driven by other tyrosine kinases including other receptor tyrosine kinases $(\mathrm{RTK})^{33,34}$. For the NCOA4-RET fusion-positive, ER+/ERBB2amplified breast cancer, one can speculate that the presence of NCOA4-RET contributed to its relative resistance to primary treatment with aromatase inhibitor, trastuzumab, and pertuzumab. While this patient had clinical benefit after switching to cabozantinib, trastuzumab, and an alternative aromatase inhibitor, one cannot determine with certainty, based on case information alone, whether that clinical benefit was primarily attributable to initiation of cabozantinib, the new aromatase inhibitor, or the combination of these drugs. Intriguingly, coexistence of NCOA4-RET with another HER family member, EGFR (HER1), has been shown as a mechanism of resistance in an EGFR-mutated lung cancer treated with afatinib ${ }^{35}$. Of note is that at least one other ERBB2-amplified breast cancer in this cohort was known to have developed RET amplification after it had acquired resistance to multiple HER2-targeted agents and that this was associated with overexpression of RET in the tumor ( ${ }^{*}$ in Fig. 1a and Supplementary Fig. 4c). Modeling of amplification by overexpression of wild-type RET sequence validates that RET overexpression, similar to RET fusions, can lead to aberrant downstream signaling like other RTKs such as ERBB2 amplification in breast cancer or MET amplification in lung cancer. The response of the RET $^{\mathrm{amp}}$ xenografts to cabozantinib also reveals the potential for RET inhibition in treating RET amplification- 
positive breast cancers although this needs to be corroborated with appropriate patient-derived xenograft models.

While interchromosomal rearrangements leading to RET fusions have been reported previously, all RET fusions detected in this cohort reflect intrachromosomal rearrangements in chromosome 10. The exclusive presence of chromosome 10 partners here may reflect loss of specific DNA damage and repair mechanisms particular to basal-like breast cancers, that have been known to exhibit numerous intrachromosomal rearrangements when compared to luminal subtypes ${ }^{36}$. However, the overriding challenge for many RET rearrangements is a need to model and characterize novel structural rearrangements to determine functionality before their identification can be incorporated appropriately for tailoring therapeutic approaches. Importantly, the RASGEF1A-RET fusion serves to model a number of novel rearrangements identified in this cohort. Other rearrangements warrant further evaluation. For instance, for four RET kinase domain duplications detected in this study (Fig. 1c), predicting ORFs with duplicated exons alone does not detect potential alternative splicing events that could lead to kinase domain duplications in the protein as algorithms recognize the native STOP codons. The recurrent finding of such duplications warrants detailed enquiry including intronic sequences and scoring of potential splice regulator sites using validated splice prediction tools. However, that there is a precedent for functional and therapeutically actionable EGFR, MET, and BRAF kinase domain duplications in other cancers is suggestive that a similar mechanism may be applicable to the observed RET kinase duplications in breast cancer ${ }^{37-39}$.

Comparison of NCOA4-RET and RASGEF1A-RET in tumorigenic and in vitro assays reveal that both fusions induce transformation of nontumorigenic cells, but NCOA4-RET potentially confers a more aggressive oncogenic phenotype than RASGEF1A$R E T$. Similar to many RET missense mutations, where the oncogenic potential may differ from variant to variant ${ }^{40}$, preclinical modeling of RET rearrangements shows differing severity of phenotypes associated with expression of the canonical CCDC6-RET and NCOA4-RET fusions ${ }^{41}$. Additionally, RET fusions that are under control of dimerizing partners (NCOA4, CCDC6) display distinctions in functional pathways employed ${ }^{41}$. This may further differentiate truncated kinases without fusion partners such as $\triangle$ RET from NCOA4-RET. The preference of one signaling pathway over another has been reported for NCOA4RET (previously referred to as RET/PTC3 in thyroid cancer) where enhanced signaling occurs via AKT rather than ERK, although both pathways are activated ${ }^{42}$. This is also observed in the model in Fig. 2e. $\triangle$ RET is modeled after RASGEF1A-RET and differential tissue expression between RET and RASGEF1A can further drive oncogenic potential by influencing expression levels of $\triangle \mathrm{RET}$, which will be under the more ubiquitous RASGEF1A promoter in the breast tissue for this fusion. Moreover, rearrangements with less oncogenic potential, such as $\triangle \mathrm{RET}$, may become more potent in the setting of specific co-events similar to synergistic potentiation by co-events observed for B-cell lymphomas ${ }^{43}$. With increasing efficiency and accuracy of genomeediting tools such as CRISPR, it is anticipated that it would become easier to assess complex genomic rearrangements at more endogenous levels of expression and in the context of tissue type.

Characterization of the novel RASGEF1A-RET ( $\triangle R E T)$ fusion revealed that an $\mathrm{N}$-terminally truncated RET kinase domain is oncogenic and targetable. Precedence for activated and targetable, $\mathrm{N}$-terminally truncated tyrosine kinases has been reported for HER2 ${ }^{44-46}, \mathrm{ALK}^{47}, \mathrm{c}-\mathrm{KIT}^{48}$, and $\mathrm{EGFR}^{49}$ which lack the extracellular and transmembrane regions in breast, melanoma, prostate cancers, and gliomas, respectively. $\triangle R E T$ also serves to model other intergenic rearrangements of RET such as those identified in this cohort that may place kinase domain-coding exons under the influence of alternate start sites, promoters, and enhancers, and could potentially render a larger group of breast cancers therapeutically actionable. The N-terminally truncated $\triangle$ RET, which includes residues $674-1072$ of RET, includes a portion of the juxtamembrane region, an intact kinase domain and the Cterminal tails. The juxtamembrane (JM) domain is functionally important in regulating auto-inhibitory functions of $\mathrm{RTKs}^{50,51}$. Loss or changes in the JM region of RET have been associated with cancer. In-frame insertion/deletion mutations and single base changes in JM domain (666 and 691) have been reported to cause gain-of-function, constitutively active, monomeric RET in familial and sporadic medullary thyroid cancer. ${ }^{21,52,53}$ With the loss of extracellular ligand-binding domain, transmembrane domain, and partial loss of the auto-inhibitory juxtamembrane domain, $\triangle \mathrm{RET}$ is hypothesized to be active by autophosphorylation in a cytosolic and ligand-independent manner.

A more comprehensive understanding of the effect of RET amplification on tumorigenesis and drug sensitivity as well as whether this alteration translates into increased mRNA and protein levels is needed, given its overall frequency in breast cancer. A recent study has demonstrated that chronic overexpression of RET wild-type sequence in an inducible, transgenic mouse model results in luminal mammary tumors that are also responsive to a RET kinase inhibitor ${ }^{54}$. The minimally amplified region around RET in this breast cancer cohort cannot be determined from targeted genomic profiling alone. Therefore, the presence of an amplicon that encompasses another oncogene surrounding RET on chromosome 10 cannot be ruled out. Notably, while $R E T$ is the only compelling oncogene from the Cancer Gene Census on the 10q11 segment, additional evaluation of this region, similar to what has been done for $8 \mathrm{p} 11$ in breast cancer, is required ${ }^{55}$. This study highlights that the frequent finding of RET amplification, its near exclusivity with respect to other RET alterations reported here, response to TKI, and the known involvement of RET overexpression in tumorigenesis and resistance to hormonal therapies is worthy of follow-up in future clinical datasets and currently available databases with access to detailed genomic, treatment, and drug response histories.

Subsets of genomic alterations in RET were enriched in breast cancer subtypes. Of all variant classes, RET amplifications were the most commonly observed and mainly found in ER - and ERBB2- (ERBB2 wild-type/HER2-) breast cancers, followed by missense mutations and rearrangements. There was a trend toward more RET rearrangements being found in ER- breast cancers. Conversely, RET missense mutations were more frequently associated with $\mathrm{ER}+$ breast cancers. RET missense mutations are found as germline mutations in cancer susceptibility syndromes such as MEN2, but may also be somatic ${ }^{56}$. Algorithms using allele frequency, tumor purity, and ploidy may suggest whether or not a missense mutation is likely germline or somatic; however, clinical care guidelines would include genetic counseling and testing based on the strength of the patient's personal medical and family history ${ }^{57}$. As genomic profiling cases may be biased toward more aggressive or refractory breast cancers and this cohort was restricted to cases with available ER status, the distribution of RET alterations may be skewed and limited in generalization to specific breast cancer subtypes. Yet, analysis of publically available breast cancer datasets through cBioPortal reveals similar trends (Supplementary Fig. 5) ${ }^{58,59}$. The presence of RET missense mutations in ER+ breast cancers, a subset which may be hormone therapy resistant, becomes highly relevant and merits further study given known associations between RET expression levels and reduced sensitivity to hormonal therapies ${ }^{9-11}$. Further studies demonstrating any differential response to specific inhibitors based on type of RET alteration may help guide use of the most appropriate inhibitor. 
Multi-kinase inhibitors with activity against RET such as sorafenib, vandetanib, and others have been tested in clinical trials in unselected breast cancers with modest to no significant activity 60,61 . However, both the presence of the target, e.g. RET alteration, and an understanding of the effect that any individual genomic alteration has on inhibitor response is critical. For instance, RET V804M is a known gatekeeper mutation that contributes to cabozantinib resistance, but tumors with this mutation may still retain sensitivity to other tyrosine kinase inhibitors with activity against RET such as ponatinib and AD80 based on preclinical data ${ }^{62,63}$. Moreover, preclinical modeling of CCDC6-RET and NCOA4-RET demonstrated distinct TKI sensitivities ${ }^{41}$. Recent clinical trials have shown that RET inhibitors are active in a subset of patients with lung cancers harboring RETrearrangements ${ }^{5,29}$. Novel approaches to target RET rearrangements are now being evaluated by developing potent inhibitors with high selectivity and improved biochemical profiles ${ }^{27,63}$. With new evidence of the presence of oncogenic, recurrent RET fusions and rearrangements in breast cancer, clinical trials catering to $R E T$-rearranged cancers need to be expanded to evaluate these selected breast cancers.

In summary, recurrent canonical and noncanonical RET alterations are observed in breast cancer. Supporting clinical considerations for targeting RET rearrangements are the functional activities attributable to the NCOA4-RET fusion and to the novel RASGEF1A-RET fusion, which are the focus of this report. Results support the oncogenic potential of both types of RET fusions as well as that of RET amplification. That RET alterations are sensitive to RET inhibitors, as demonstrated in vitro and in vivo models as well as in a patient, suggest that they are potentially actionable in the subset of breast cancers in which they are found. Further studies are warranted to better understand the frequency and correlation of RET alterations with response to standard therapies, and the ideal strategies to therapeutically target RET and improve patient outcomes.

\section{Methods \\ Study population. Formalin-fixed, paraffin-embedded (FFPE) tissue samples} obtained from a total available $n=9693$ patients with breast cancer were submitted by clinicians for targeted next-generation sequencing as part of routine clinical care. The pathologic diagnosis of each submitted case was confirmed and tumor content was determined on routine hematoxylin- and eosin-stained slides. Specimens were submitted with limited accompanying clinical information; pathology reports were reviewed to determine ER status where available. Approval for this study, including a waiver of informed consent and an HIPAA (Health Insurance Portability and Accountability Act of 1996) waiver of authorization, was obtained from the Western Institutional Review Board (Protocol No. 20152817). The study complied with all ethical regulations relevant to human research participants and data.

DNA and RNA sequencing. Targeted next-generation sequencing was performed in a Clinical Laboratory Improvement Amendments (CLIA)-certified, New York State-accredited, and College of American Pathologists (CAP)-accredited laboratory (Foundation Medicine, Inc., Cambridge, MA) between May 2012 and Sept. 2016. Technical details and validation of genomic profiling assays have been extensively described ${ }^{64,65}$. In brief, at least $50 \mathrm{ng}$ DNA was extracted from $40-\mu \mathrm{m}$ FFPE sections with at least $20 \%$ tumor cells. Adaptor-ligated DNA underwent hybrid capture for all coding exons of 236, 315, or 405 cancer-related genes plus select introns from 19, 28, or 31 genes frequently rearranged in cancer (Supplementary Data 1 for gene list). Captured libraries were sequenced to a median exon coverage depth of $637 \times$ (Illumina HiSeq). Resultant sequences were analyzed for short variants (base substitutions, insertions/deletions (indels)), copy number alterations (focal amplifications and homozygous deletions), and select gene fusions/rearrangements. RNA sequences were analyzed for the presence of fusions/ rearrangements only. Custom filtering was applied to report genomic alterations and remove benign germline events ${ }^{64,65}$. Copy number detection and validation was obtained by normalizing the reads in the tumor against a process-matched normal control and further corrected for tumor purity ${ }^{64}$. Germline versus somatic variant status for missense mutations were determined based on allele frequencies, altered copy numbers, and tumor purity ${ }^{24,25}$.
Fusion sequences and expression plasmids. The human fusion ORF cDNA sequences for NCOA4 were amplified from MCF7 (ATCC ${ }^{\circledast}$ HTB-22 ${ }^{\text {"x }}$ ) cell line and $\triangle R E T$ was amplified from pDONR223-RET, a gift from William Hahn \& David Root (Addgene plasmid \#23906) using recombinant Taq DNA polymerase enzyme (Life Technologies) with fusion-specific primers (Supplementary Table 3). Gelextracted PCR products for NCOA4-RET were ligated using T4 DNA ligase enzyme (Invitrogen). The predicted ORFs for NCOA4-RET, $\triangle R E T$ and full-length, wildtype RET (RET ${ }^{a m p}$ ) were cloned into pLenti6.3/V5-DEST and pEF-DEST51 vectors (Invitrogen) that include a C-terminal V5-tag. Constitutively kinase active (corresponding to M918T) and kinase inactive (corresponding to K758M) fulllength variants were generated using QuikChange II XL Site-Directed Mutagenesis Kit (Agilent). All constructs were verified by DNA sequencing.

Cell lines and in vitro overexpression of ORFs. Human breast cell line MCF 10A (ATCC ${ }^{\circledR}$ CRL-10317 ${ }^{\text {Tx }}$ ) and mouse fibroblast cell line NIH/3T3 (ATCC ${ }^{\circledR}$ CRL$1658^{\mathrm{si}}$ ) were obtained from American Type Culture Collection (ATCC) and cultured according to ATCC recommended protocols. Authentication of all cell lines was performed by ATCC using short tandem repeat analysis and mycoplasma contamination tested negative. Both MCF10A and NIH/3T3 cells lacked mRNA expression of RET co-receptor, GFRa as verified by qPCR and detectable RET protein as measured by western blot. The pLenti6.3 vectors were used for overexpression in MCF10a using Fugene HD transfection (Promega) and in NIH/3T3 cells using Lipofectamine 3000 (Invitrogen). pEF-DEST51 vectors were transduced into NIH/3T3 and selected with blasticidin (Invitrogen). For all cell line experiments and growth curves, cells were counted using Vi-CELL XR (Beckman Coulter) cell analyzer using trypan blue die exclusion method. For measurements involving phosphorylated proteins, NIH/3T3 and MCF10A cells were serum starved for $24 \mathrm{~h}$ in DMEM media before protein isolation, which is described in detail below. For all other experiments, NIH/3T3 cells were cultured with $10 \%$ bovine calf serum in media and MCF10A cells were cultured in ATCCrecommended mammary epithelial growth media that includes mammary epithelial basal media supplemented with bovine pituitary extract, human epidermal growth factor, insulin and hydrocortisone (MEGM-kit, Lonza, CC-3150).

Western blot analysis. Protein samples were extracted from cells and tumor xenografts using NETN lysis buffer (Tris $20 \mathrm{mM} \mathrm{pH} \mathrm{8.0,} \mathrm{NaCl} 150 \mathrm{mM} \mathrm{pH} \mathrm{7.5,}$ EDTA $1 \mathrm{mM}$, NP40 0.5\%) along with protease and phosphatase inhibitor cocktails (Sigma-Aldrich). For lysates from xenografts tumors, frozen tissue was weighed and approximately $20 \mathrm{mg}$ was homogenized in $200 \mu \mathrm{l}$ lysis buffer on ice. After homogenization, samples were briefly sonicated twice for $20 \mathrm{~s}$ each and incubated on ice for $30 \mathrm{~min}$, followed by centrifugation at $14,000 \mathrm{rpm}$ for $15 \mathrm{~min}$ at $4{ }^{\circ} \mathrm{C}$. For lysates from cells, samples were vortexed for $5 \mathrm{~s}$ every 5 min while incubating on ice for $15 \mathrm{~min}$. This was followed by centrifugation at $14,000 \mathrm{rpm}$ for $10 \mathrm{~min}$ at $4{ }^{\circ} \mathrm{C}$. All lysates were separated by gradient (4-12\%) sodium dodecyl sulphatepolyacrylamide gel electrophoresis (SDS-PAGE) under reducing conditions and transferred onto $0.2 \mu \mathrm{m}$ polyvinylidene fluoride membrane for detection. The dilutions of primary antibodies used were 1:250-1:1000 for anti-phospho-Ret (Y905) (Cell Signaling Technology, 3221), 1:250 for anti-phospho-Ret (Y1062) (Santa Cruz Biotechnology, 20252-R) and 1:1000 for anti-Ret specific to the Cterminal region (Cell Signaling Technology, 14698). For all other rabbit primary antibodies (phospho and total MEK $(9154,9126)$, P70 S6 $(9234,2708)$ and total V5-tag (13202)), dilutions were used at 1:1000 (Cell Signaling Technology). Mouse anti-actin was used at 1:10,000 (Sigma-Aldrich, clone AC-15). The full list of antibodies and details are included in Supplementary Table 4. Enhanced chemiluminescence (ECL) reagent was added to the membrane (Pierce Biotechnology) and bands were detected on the ChemiDoc ${ }^{\mathrm{Tm}}$ Imaging System. Image densitometry was performed using the Image Lab software (Bio-Rad). For analyzing fusiondriven signaling, cells were serum-starved for $24 \mathrm{~h}$ prior to protein extraction. To study changes in fusion signaling under the influence of RET inhibitor cabozantinib (Selleckchem), cells were serum-starved overnight and treated with cabozantinib for $1 \mathrm{~h}$ and compared to vehicle DMSO-treated controls. Uncropped scans of western blot images in Figs. 2 and 3 are shown in Supplementary Figs. 6-7.

Cell viability assay. Cell viability was measured using the CellTiter Aqueous MTS reagent (Promega) according to the manufacturer's protocol. Following optimization and accounting for growth rate differences, 1000 NCOA4-RET cells and 2000 vector, $R T^{\mathrm{amp}}$ and $\triangle \mathrm{RET}$ cells were allowed to attach per well in 96-well plates overnight for NIH/3T3 cells. For MCF10A, all cells were plated at 5000 per well. Cells were then treated with increasing concentration of inhibitors, cabozantinib or sorafenib (Selleckchem) for $72 \mathrm{~h}$. After incubating with MTS reagent for $2-4 \mathrm{~h}$ at $37^{\circ} \mathrm{C}$, absorbance was measured at $490 \mathrm{~nm}$ on a plate reader (Infinite 200 PRO, Tecan). Viability of cells was calculated by dividing the average absorbance of treatment wells by that of vehicle DMSO-treated wells. Data were analyzed and represented using GraphPad Prism version 7 software.

Clonogenic assay. Cells were seeded at 150 per well in six-well plates and incubated for 14 days. Colonies were stained with crystal violet ( $2 \%$ solution, SigmaAldrich) and visualized using ChemiDoc ${ }^{\mathrm{m} x}$ Imaging System (Bio-Rad). Colonies greater than 50 cells were counted using a cell counter (ImageJ) for comparison 
between groups. For experiments involving inhibitor treatment, cells were incubated with inhibitor (cabozantinib, vandetanib (Selleckchem)) or DMSO in media for 14 days prior to crystal violet staining.

Animal xenografts study and immunohistochemistry. All experiments were performed under the approval of the Rutgers University Institutional Animal Care and Use Committee and complied with all relevant ethical regulations. Based on the experiment, $0.5,1,4.5$ or $5 \times 10^{6}$ transduced $\mathrm{NIH} / 3 \mathrm{~T} 3$ cells were suspended in a 1:1 ratio in high concentration Matrigel basement membrane matrix (Corning) and injected subcutaneously in 6-8-week-old, female, athymic nude mice (Taconic, CrTac:NCrFoxn1nu (NCr Nude) or NOD/SCID/interleukin 2 receptor $\gamma$ null mice (The Jackson Laboratory, NOD.Cg-Prkdcscid Il2rgtmlWjl /SzJ (NSG)). For tumor formation, $1 \times 10^{6}$ cells were injected for all three RET altered cell lines in nude mice. For RET ${ }^{\text {amp }}$, tumor formation was further examined at $4.5 \times 10^{6}$ cells in NSG mice. Tumors were monitored twice weekly by palpation and tumor dimensions were measured using digital calipers (VWR). Tumor volumes were calculated using the formula $1 / 2\left(\right.$ length $\times$ width $\left.^{2}\right)$. For drug treatment, $0.5 \times 10^{6}$ NCOA4-RET cells and $5 \times 10^{6} \Delta$ RET and RET Tamp cells were injected bilaterally into flanks of athymic nude mice and were allowed to form palpable tumors before randomization. Allocation to treatment groups ( $n=5$ for NCOA4-RET and $n=7$ for $\triangle \mathrm{RET}$ and RET ${ }^{\text {amp }}$ studies per group) was performed when average tumor volumes measured about $300 \mathrm{~mm}^{3}$ using simple randomization with stratification and blinding to group allocation. Cabozantinib was dosed orally at 30 or $60 \mathrm{mg} \mathrm{kg}^{-1}$ once daily for 14 days and was formulated according to the manufacturer's specifications. Tumor measurements and body weights were recorded three times a week for each animal upon initiating treatment. Immunohistochemical staining was prepared using a Ventana XT Discovery automated staining device according to the manufacturer's protocol. Primary antibodies Ki-67 (Spring Biosciences, M3062), Cleaved Caspase-3 (Cell Signaling Technology, 9661) were used at 1:400 and 1:200 dilutions respectively (Supplementary Table 4).

Statistical analysis. All experiments including three or more groups were analyzed using one-way ANOVA or two-way ANOVA for two variables. Multiple comparisons were performed using Tukey's test. All data represented as mean \pm s.d. $p$ value $\leq 0.05$ was considered statistically significant in all experiments. Analyses were performed using Prism, version 7.0d (GraphPad software).

Reporting summary. Further information on research design is available in the Nature Research Reporting Summary linked to this article.

\section{Data availability}

The authors declare that the data supporting the findings in the study are available in the main article and supplementary files or available from corresponding authors upon request. A reporting summary for this article is available as a Supplementary Information file.

Received: 2 January 2018 Accepted: 25 October 2018

Published online: 16 November 2018

\section{References}

1. Agrawal, N. et al. Integrated genomic characterization of papillary thyroid carcinoma. Cell 159, 676-690 (2014)

2. Wang, R. et al. RET fusions define a unique molecular and clinicopathologic subtype of non-small-cell lung cancer. J. Clin. Oncol. 30, 4352-4359 (2012).

3. Mulligan, L. M. RET revisited: expanding the oncogenic portfolio. Nat. Rev. Cancer 14, 173-186 (2014).

4. Richardson, D. S., Gujral, T. S., Peng, S., Asa, S. L. \& Mulligan, L. M. Transcript level modulates the inherent oncogenicity of RET/PTC oncoproteins. Cancer Res. 69, 4861-4869 (2009).

5. Drilon, A. et al. Cabozantinib in patients with advanced RET-rearranged nonsmall-cell lung cancer: an open-label, single-centre, phase 2, single-arm trial. Lancet Oncol. 17, 1653-1660 (2016).

6. Lee, S.-H. et al. A phase II study of vandetanib in patients with non-small cell lung cancer harboring RET rearrangement. J. Clin. Oncol. 34, 9013-9013 (2016).

7. Esseghir, S. et al. A role for glial cell derived neurotrophic factor induced expression by inflammatory cytokines and RET/GFR alpha 1 receptor upregulation in breast cancer. Cancer Res. 67, 11732-11741 (2007).

8. Hatem, R. et al. Vandetanib as a potential new treatment for estrogen receptor-negative breast cancers. Int. J. Cancer 138, 2510-2521 (2016).

9. Morandi, A. et al. GDNF-RET signaling in ER-positive breast cancers is a key determinant of response and resistance to aromatase inhibitors. Cancer Res. 73, 3783-3795 (2013).

10. Spanheimer, P. M. et al. Distinct pathways regulated by RET and estrogen receptor in luminal breast cancer demonstrate the biological basis for combination therapy. Ann. Surg. 259, 793-799 (2014).
11. Spanheimer, P. M. et al. Inhibition of RET increases the efficacy of antiestrogen and is a novel treatment strategy for luminal breast cancer. Clin. Cancer Res. 20, 2115-2125 (2014).

12. Griseri, P. et al. Genetic and epigenetic factors affect RET gene expression in breast cancer cell lines and influence survival in patients. Oncotarget 7 , 26465-26479 (2016).

13. Andreucci, E. et al. Targeting the receptor tyrosine kinase RET in combination with aromatase inhibitors in ER positive breast cancer xenografts. Oncotarget 7, 80543-80553 (2016).

14. Paratala, B. S. et al. Emerging role of genomic rearrangements in breast cancer: applying knowledge from other cancers. Biomark. Cancer 8, 1-14 (2016).

15. Tognon, C. et al. Expression of the ETV6-NTRK3 gene fusion as a primary event in human secretory breast carcinoma. Cancer Cell. 2, 367-376 (2002).

16. Veeraraghavan, J. et al. Recurrent ESR1-CCDC170 rearrangements in an aggressive subset of oestrogen receptor-positive breast cancers. Nat. Commun. 5, 4577 (2014).

17. Monaco, C. et al. The RFG oligomerization domain mediates kinase activation and re-localization of the RET/PTC3 oncoprotein to the plasma membrane. Oncogene 20, 599-608 (2001).

18. Takahashi, M., Ritz, J. \& Cooper, G. M. Activation of a novel human transforming gene, ret, by DNA rearrangement. Cell 42, 581-588 (1985).

19. Tong, Q., Xing, S. \& Jhiang, S. M. Leucine zipper-mediated dimerization is essential for the PTC1 oncogenic activity. J. Biol. Chem. 272, 9043-9047 (1997).

20. Gainor, J. F. \& Shaw, A. T. The new kid on the block: RET in lung cancer. Cancer Discov. 3, 604-606 (2013).

21. Muzza, M. et al. Four novel RET germline variants in exons 8 and 11 display an oncogenic potential in vitro. Eur. J. Endocrinol. 162, 771-777 (2010).

22. Silva, A. L. et al. Identification and characterization of two novel germline RET variants associated with medullary thyroid carcinoma. Endocrine 49, 366-372 (2015).

23. Forbes, S. A. et al. COSMIC: somatic cancer genetics at high-resolution. Nucleic Acids Res. 45, D777-D783 (2017).

24. Sun, J. X. et al. A computational approach to distinguish somatic vs. germline origin of genomic alterations from deep sequencing of cancer specimens without a matched normal. PLoS Comput. Biol. 14, el 005965 (2018).

25. Khiabanian, $\mathrm{H}$. et al. Inference of germline mutational status and evaluation of loss of heterozygosity in high-depth tumor-only sequencing data. JCO Precis. Oncol. https://doi.org/10.1200/PO.17.00148 (2018).

26. Okamoto, K. et al. Antitumor activities of the targeted multi-tyrosine kinase inhibitor lenvatinib (E7080) against RET gene fusion-driven tumor models. Cancer Lett. 340, 97-103 (2013).

27. Li, G. G. et al. Antitumor activity of RXDX-105 in multiple cancer types with RET rearrangements or mutations. Clin. Cancer Res. 23, 2981-2990 (2017).

28. Sabari, J. K., Siau, E. D. \& Drilon, A. Targeting RET-rearranged lung cancers with multikinase inhibitors. Oncoscience 4, 23-24 (2017).

29. Yoh, K. et al. Vandetanib in patients with previously treated RET-rearranged advanced non-small-cell lung cancer (LURET): an open-label, multicentre phase 2 trial. Lancet Respir. Med. 5, 42-50 (2017).

30. Drilon, A. et al. Response to Cabozantinib in patients with RET fusionpositive lung adenocarcinomas. Cancer Discov. 3, 630-635 (2013).

31. DeSantis, C. E., Ma, J., Goding Sauer, A., Newman, L. A. \& Jemal, A. Breast cancer statistics, 2017, racial disparity in mortality by state. CA Cancer J. Clin. 67, 439-448 (2017).

32. Soda, M. et al. Identification of the transforming EML4-ALK fusion gene in non-small-cell lung cancer. Nature 448, 561-566 (2007).

33. Ali, S. M. et al. A combination of targeted therapy with chemotherapy backbone induces response in a treatment-resistant triple-negative MCL1amplified metastatic breast cancer patient. Case Rep. Oncol. 9, 112-118 (2016).

34. Kulkarni, A. et al. BRAF fusion as a novel mechanism of acquired resistance to Vemurafenib in BRAFV600E mutant melanoma. Clin. Cancer Res. 23, 5631-5638 (2017)

35. Klempner, S. J. et al. Emergence of RET rearrangement co-existing with activated EGFR mutation in EGFR-mutated NSCLC patients who had progressed on firstor second-generation EGFR TKI. Lung Cancer 89, 357-359 (2015).

36. Kwei, K. A., Kung, Y., Salari, K., Holcomb, I. N. \& Pollack, J. R. Genomic instability in breast cancer: pathogenesis and clinical implications. Mol. Oncol. 4, 255-266 (2010)

37. Baik, C. S., Wu, D., Smith, C., Martins, R. G. \& Pritchard, C. C. Durable response to tyrosine kinase inhibitor therapy in a lung cancer patient harboring epidermal growth factor receptor tandem kinase domain duplication. J. Thorac. Oncol. 10, e97-e99 (2015).

38. Klempner, S. J. et al. Identification of BRAF kinase domain duplications across multiple tumor types and response to RAF inhibitor therapy. JAMA Oncol. 2, 272-274 (2016)

39. Plenker, D. et al. Structural alterations of MET trigger response to MET kinase inhibition in lung adenocarcinoma patients. Clin. Cancer Res. 24, 1337-1343 (2018). 
40. Frank-Raue, K., Rondot, S. \& Raue, F. Molecular genetics and phenomics of RET mutations: impact on prognosis of MTC. Mol. Cell. Endocrinol. 322, 2-7 (2010).

41. Levinson, S. \& Cagan, R. L. Drosophila cancer models identify functional differences between Ret fusions. Cell Rep. 16, 3052-3061 (2016).

42. Miyagi, E. et al. Chronic expression of RET/PTC 3 enhances basal and insulinstimulated PI3 kinase/AKT signaling and increases IRS-2 expression in FRTL5 thyroid cells. Mol. Carcinog. 41, 98-107 (2004).

43. Nakagawa, M., Tsuzuki, S., Honma, K., Taguchi, O. \& Seto, M. Synergistic effect of Bcl2, Myc and Ccnd1 transforms mouse primary B cells into malignant cells. Haematologica 96, 1318-1326 (2011).

44. Christianson, T. A. et al. NH2-terminally truncated HER-2/neu protein: relationship with shedding of the extracellular domain and with prognostic factors in breast cancer. Cancer Res. 58, 5123-5129 (1998).

45. Molina, M. A. et al. NH2-terminal truncated HER-2 protein but not fulllength receptor is associated with nodal metastasis in human breast cancer. Clin. Cancer Res. 8, 347-353 (2002).

46. Scaltriti, M. et al. Expression of p95HER2, a truncated form of the HER2 receptor, and response to anti-HER2 therapies in breast cancer. J. Natl. Cancer Inst. 99, 628-638 (2007).

47. Wiesner, T. et al. Alternative transcription initiation leads to expression of a novel ALK isoform in cancer. Nature 526, 453-457 (2015).

48. Paronetto, M. P. et al. Expression of a truncated form of the c-Kit tyrosine kinase receptor and activation of Src kinase in human prostatic cancer. Am. J. Pathol. 164, 1243-1251 (2004).

49. Huang, P. H., Xu, A. M. \& White, F. M. Oncogenic EGFR signaling networks in glioma. Sci. Signal. 2, 87 pp re6 (2009).

50. Chan, P. M., Ilangumaran, S., La Rose, J., Chakrabartty, A. \& Rottapel, R. Autoinhibition of the kit receptor tyrosine kinase by the cytosolic juxtamembrane region. Mol. Cell. Biol. 23, 3067-3078 (2003).

51. Runeberg-Roos, P. \& Saarma, M. Neurotrophic factor receptor RET: structure, cell biology, and inherited diseases. Ann. Med. 39, 572-580 (2007).

52. Borrello, M. G. et al. Functional characterization of the MTC-associated germline RET-K666E mutation: evidence of oncogenic potential enhanced by the G691S polymorphism. Endocr. Relat. Cancer 18, 519-527 (2011).

53. Cordella, D. et al. An in-frame complex germline mutation in the juxtamembrane intracellular domain causing RET activation in familial medullary thyroid carcinoma. Endocr. Relat. Cancer 13, 945-953 (2006).

54. Gattelli, A. et al. Chronic expression of wild-type Ret receptor in the mammary gland induces luminal tumors that are sensitive to Ret inhibition. Oncogene 37, 4046-4054 (2018).

55. Ray, M. E. et al. Genomic and expression analysis of the 8p11-12 amplicon in human breast cancer cell lines. Cancer Res. 64, 40-47 (2004).

56. Arighi, E., Borrello, M. G. \& Sariola, H. RET tyrosine kinase signaling in development and cancer. Cytokine Growth Factor Rev. 16, 441-467 (2005).

57. Hirshfield, K. M. et al. Clinical actionability of comprehensive genomic profiling for management of rare or refractory cancers. Oncologist $\mathbf{2 1}$, 1315-1325 (2016).

58. Cerami, E. et al. The cBio cancer genomics portal: an open platform for exploring multidimensional cancer genomics data. Cancer Discov. 2, 401 (2012).

59. Gao, J. et al. Integrative analysis of complex cancer genomics and clinical profiles using the cBioPortal. Sci. Signal. 6, pl1 (2013).

60. Bronte, G. et al. Sorafenib for the treatment of breast cancer. Expert Opin. Pharmacother. 18, 621-630 (2017).

61. Miller, K. D. et al. A multicenter phase II trial of ZD6474, a vascular endothelial growth factor receptor-2 and epidermal growth factor receptor tyrosine kinase inhibitor, in patients with previously treated metastatic breast cancer. Clin. Cancer Res. 11, 3369-3376 (2005).

62. Mologni, L., Redaelli, S., Morandi, A., Plaza-Menacho, I. \& GambacortiPasserini, C. Ponatinib is a potent inhibitor of wild-type and drug-resistant gatekeeper mutant RET kinase. Mol. Cell. Endocrinol. 377, 1-6 (2013).

63. Plenker, D. et al. Drugging the catalytically inactive state of RET kinase in RET-rearranged tumors. Sci. Transl. Med. 9, eaah6144 (2017).
64. Frampton, G. M. et al. Development and validation of a clinical cancer genomic profiling test based on massively parallel DNA sequencing. Nat. Biotechnol. 31, 1023-1031 (2013).

65. Hartmaier, R. J. et al. High-throughput genomic profiling of adult solid tumors reveals novel insights into cancer pathogenesis. Cancer Res. 77, 2464-2475 (2017).

\section{Acknowledgements}

The authors acknowledge the editorial, technical, and administrative assistance of Susan Moench, Jennifer Hostettler, and Jacqueline Harris. This work was supported by the AHEPA 5th District Cancer Research Foundation, the Stacy Goldstein Faculty Scholar Award established by Suzann and Edwin Goldstein (K.M.H.), National Institutes of Health Grant P30CA072720, through a generous gift to the Genetics Diagnostics to Cancer Treatment Program of the Rutgers Cancer Institute of New Jersey and Rutgers University Cell and DNA Repository (RUCDR) Infinite Biologics, Shared Resources of the Rutgers Cancer Institute of New Jersey including the Biospecimen Repository Service, Histopathology and Imaging, and Office for Human Research Service.

\section{Author contributions}

K.M.H., B.L.-J., and S.M.A. conceived and supervised the study. B.S.P. designed, carried out experiments. B.S.P and J.H.C performed data analysis. W.P., K.P., S.L., and M.Y. carried out experiments. B.S.P., K.M.H. and J.H.C. co-wrote the manuscript. S.G. and A. K. advised on the study. B.L.-J., C.B.W., and K.W. gathered and contributed to clinical case data. A.B.S., L.M.G., F.D., and E.L. collected and analyzed data. J.S.R. and C.L. provided pathological analysis. B.Y. and J.H.C. conducted bioinformatics analysis. S.C.D., F.D., L.R.-R. and S.G. edited and revised the manuscript. All authors discussed the results and implications and commented on the manuscript at all stages.

\section{Additional information}

Supplementary Information accompanies this paper at https://doi.org/10.1038/s41467 018-07341-4.

Competing interests: J.H.C., B.Y., A.B.S., L.M.G., J.S.R., and S.M.A. are employees of and own stock in Foundation Medicine, Inc. K.M.H. is now an employee of and owns stock in Merck. The remaining authors declare no competing interests.

Reprints and permission information is available online at http://npg.nature.com/ reprintsandpermissions/

Publisher's note: Springer Nature remains neutral with regard to jurisdictional claims in published maps and institutional affiliations.

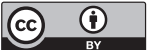

Open Access This article is licensed under a Creative Commons Attribution 4.0 International License, which permits use, sharing, adaptation, distribution and reproduction in any medium or format, as long as you give appropriate credit to the original author(s) and the source, provide a link to the Creative Commons license, and indicate if changes were made. The images or other third party material in this article are included in the article's Creative Commons license, unless indicated otherwise in a credit line to the material. If material is not included in the article's Creative Commons license and your intended use is not permitted by statutory regulation or exceeds the permitted use, you will need to obtain permission directly from the copyright holder. To view a copy of this license, visit http://creativecommons.org/ licenses/by/4.0/.

(c) The Author(s) 2018 